\title{
Retrofitting Municipal Wastewater Treatment Facilities towards Greener and Circular Economy by Virtue of Resource Recovery: Techno-economic Analysis and Life Cycle Assessment
}

\author{
Xueyu $\operatorname{Tian}^{1}$, Ruth E. Richardson ${ }^{2,3}$, Jefferson W. Tester ${ }^{1,2,4,5}$, \\ José L. Lozano ${ }^{6}$, Fengqi You ${ }^{1,2,3,4,5^{*}}$ \\ ${ }^{1}$ Systems Engineering, College of Engineering, \\ Cornell University, Ithaca, New York, 14853, USA \\ ${ }^{2}$ Atkinson Centre for a Sustainable Future, \\ Cornell University, Ithaca, New York, 14853, USA \\ 3 School of Civil and Environmental Engineering, \\ Cornell University, Ithaca, New York, 14853, USA \\ ${ }^{4}$ Robert Frederick Smith School of Chemical and Biomolecular Engineering, \\ Cornell University, Ithaca, New York, 14853, USA \\ ${ }^{5}$ Cornell Energy Systems Institute, \\ Cornell University, Ithaca, New York, 14853, USA \\ ${ }^{6}$ Environmental Laboratory, Ithaca Area Wastewater Treatment Facility, \\ 525 Third Street, Ithaca, New York, 14850, USA
}

This file contains: 61 pages, 5 sections, 4 figures, and 44 tables.

* Corresponding author. Phone: (607) 255-1162; Fax: (607) 255-9166; E-mail: fengqi.you@cornell.edu 


\section{List of Sections}

Section S1. Midpoint environmental impact score breakdowns of the reference design and retrofits of WWTF

Section S2. Key input parameters used in CapdetWorks and capital cost estimates for the reference design.

Section S3. Key input parameters used in CapdetWorks and capital cost estimates for the retrofitting design with hydrothermal liquefaction.

Section S4. Key input parameters used in CapdetWorks and capital cost estimates for the retrofitting design with pyrolysis.

Section S5. NPV calculations.

\section{List of Figures}

Figure S1. Environmental profile of the reference design on a logarithmic scale.

Figure S2. Environmental profile of the retrofitting design with HTL on a logarithmic scale.

Figure S3. Environmental profile of the retrofitting design with slow pyrolysis on a logarithmic scale.

Figure S4. Environmental profile of the retrofitting design with fast pyrolysis on a logarithmic scale.

\section{List of Tables}

Table S1. Summary of Air Supply System for the reference design.

Table S2. Summary of Chemical Feed System for Iron for the reference design.

Table S3. Summary of Influent Pump Station for the reference design.

Table S4. Summary of Preliminary Treatment for the reference design.

Table S5. Summary of Primary Clarification for the reference design.

Table S6. Summary of Anaerobic Digestion for the reference design.

Table S7. Summary of Belt-Filter Press for the reference design.

Table S8. Summary of Plug Flow Activated Sludge for the reference design.

Table S9. Summary of Gravity Thickening for the reference design.

Table S10. Summary of Hauling and Land Filling for the reference design.

Table S11. Summary of Secondary Clarifier for the reference design. 
Table S12. Summary of Chemical Phosphorus Removal for the reference design.

Table S13. Summary of Tertiary Clarification for the reference design.

Table S14. Summary of Chlorination for the reference design.

Table S15. Summary of Air Supply System for the retrofitting design with hydrothermal liquefaction.

Table S16. Summary of Chemical Feed System for Iron for the retrofitting design with hydrothermal liquefaction.

Table S17. Summary of Chemical Feed System for Lime for the retrofitting design with hydrothermal liquefaction.

Table S18. Summary of Influent Pump Station for the retrofitting design with hydrothermal liquefaction.

Table S19. Summary of Preliminary Treatment for the retrofitting design with hydrothermal liquefaction.

Table S20. Summary of Counter Current Ammonia Stripping for the retrofitting design with hydrothermal liquefaction.

Table S21. Summary of Primary Clarification for the retrofitting design with hydrothermal liquefaction.

Table S22. Summary of Anaerobic Digestion for the retrofitting design with hydrothermal liquefaction.

Table S23. Summary of Plug Flow Activated Sludge for the retrofitting design with hydrothermal liquefaction.

Table S24. Summary of Gravity Thickening for the retrofitting design with hydrothermal liquefaction.

Table S25. Summary of Secondary Clarifier for the retrofitting design with hydrothermal liquefaction.

Table S26. Summary of Chemical Phosphorus Removal for the retrofitting design with hydrothermal liquefaction.

Table S27. Summary of Tertiary Clarification for the retrofitting design with hydrothermal liquefaction.

Table S28. Summary of Chlorination for the retrofitting design with hydrothermal liquefaction.

Table S29. Summary of Air Supply System for the retrofitting design with pyrolysis. 
Table S30. Summary of Chemical Feed System for Lime for the retrofitting design with pyrolysis. Table S31. Summary of Influent Pump Station for the retrofitting design with pyrolysis.

Table S32. Summary of Preliminary Treatment for the retrofitting design with pyrolysis.

Table S33. Summary of Upflow Anaerobic Sludge Blanket Reactor for the retrofitting design with pyrolysis.

Table S34. Summary of Counter Current Ammonia Stripping for the retrofitting design with pyrolysis.

Table S35. Summary of Anaerobic Digestion for the retrofitting design with pyrolysis.

Table S36. Summary of Plug Flow Activated Sludge for the retrofitting design with pyrolysis.

Table S37. Summary of Gravity Thickening for the retrofitting design with pyrolysis.

Table S38. Summary of Belt-Filter Press for the retrofitting design with pyrolysis.

Table S39. Summary of Secondary Clarifier for the retrofitting design with pyrolysis.

Table S40. Summary of Chlorination for the retrofitting design with pyrolysis.

Table S41. NPV calculation for the reference design.

Table S42. NPV calculation for the retrofitting design with HTL.

Table S43. NPV calculation for the retrofitting design with slow pyrolysis.

Table S44. NPV calculation for the retrofitting design with fast pyrolysis. 


\section{S1. Midpoint environmental impact score breakdowns of the reference design and retrofits of WWTF}

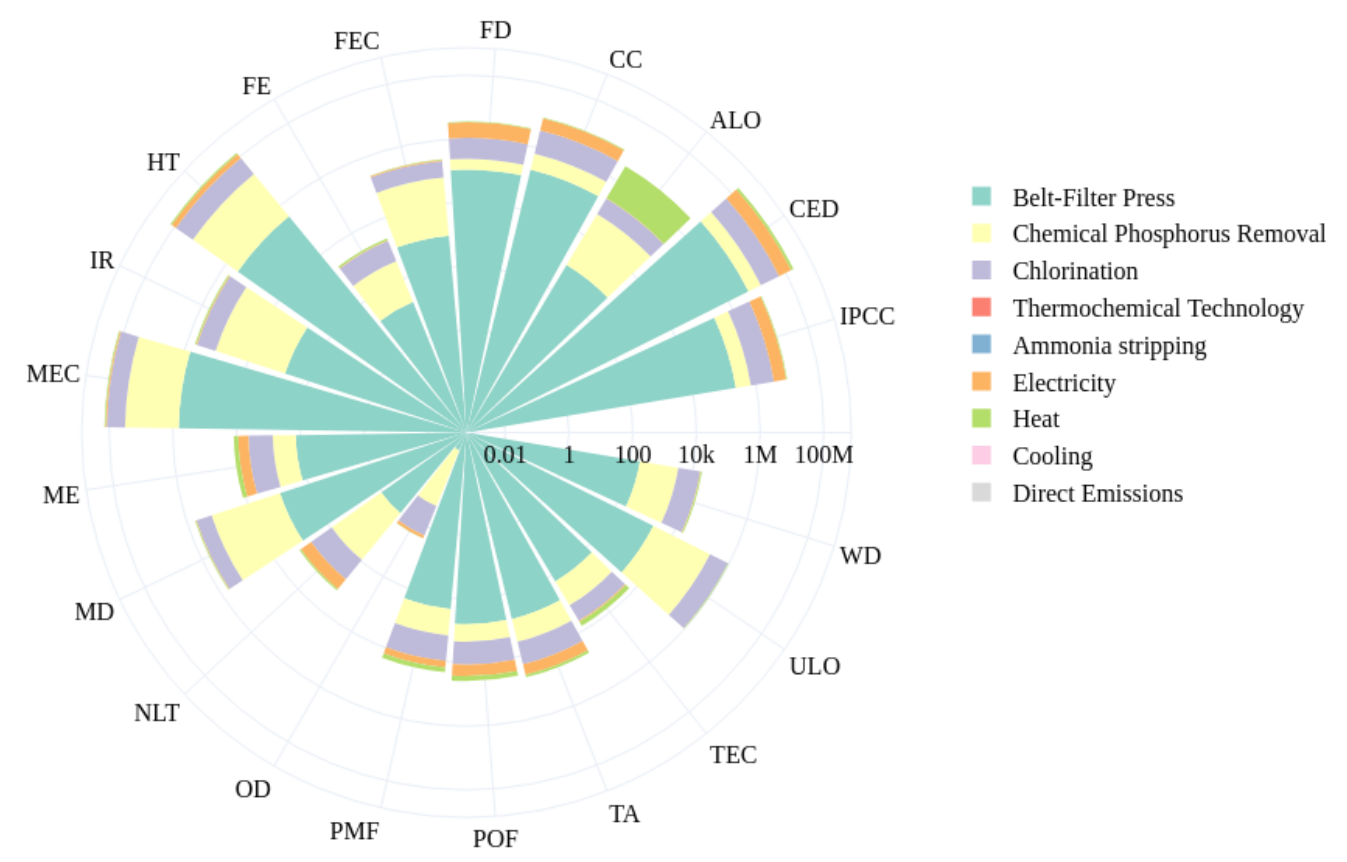

Figure S1. Environmental profile of the reference design on a logarithmic scale. The radial axis indicates the absolute quantity of each midpoint impact category, where "k" refers to one thousand and "M" stands for one million. Acronyms go counter clockwise: global warming potential (GWP) according to IPCC (IPCC, $\mathrm{kg} \mathrm{CO}_{2}$-Eq), cumulative energy demand (CED, MJ$\mathrm{Eq}$ ), agricultural land occupation (ALO, $\mathrm{m}^{2} \mathrm{a}$ ), climate change (CC, $\mathrm{kg} \mathrm{CO}_{2}-\mathrm{Eq}$ ), fossil fuel depletion (FD, kg oil-Eq), freshwater ecotoxicity (FEC, kg 1,4-DCB-Eq), freshwater eutrophication (FE, kg P-Eq), human toxicity (HT, kg 1,4-DCB-Eq), ionizing radiation (IR, kg U235-Eq), marine ecotoxicity (MEC, kg 1,4-DCB-Eq), marine eutrophication (ME, kg N-Eq), mineral resource depletion (MD, kg Fe-Eq), natural land transformation (NLT, m²), ozone depletion (OD, kg CFC-11-Eq), particulate matter formation (PMF, kg PM10-Eq), photochemical oxidant formation (POF, kg NMVOC), terrestrial acidification (TA, $\mathrm{kg} \mathrm{SO}_{2}$-Eq), terrestrial ecotoxicity (TEC, kg 1,4-DCB-Eq), urban land occupation (ULO, $\mathrm{m}^{2} \mathrm{a}$ ), water depletion (WD, $\mathrm{m}^{3}$ ). 


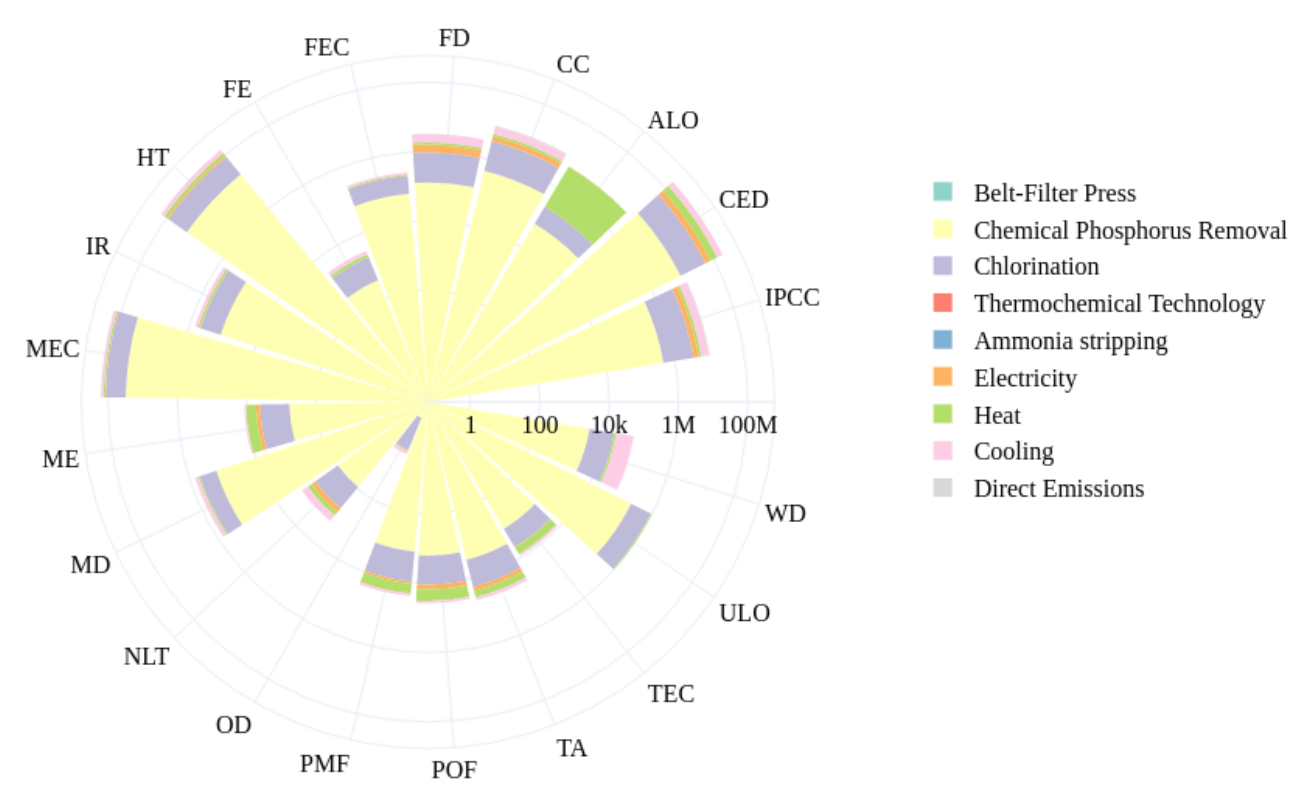

Figure S2. Environmental profile of the retrofitting design with HTL on a logarithmic scale. The radial axis indicates the absolute quantity of each midpoint impact category, where "k" refers to one thousand and "M" stands for one million. Acronyms go counter clockwise: global warming potential (GWP) according to IPCC (IPCC, $\mathrm{kg} \mathrm{CO}_{2}$-Eq), cumulative energy demand (CED, MJ$\mathrm{Eq}$ ), agricultural land occupation (ALO, $\mathrm{m}^{2} \mathrm{a}$ ), climate change (CC, $\mathrm{kg} \mathrm{CO}_{2}-\mathrm{Eq}$ ), fossil fuel depletion (FD, kg oil-Eq), freshwater ecotoxicity (FEC, kg 1,4-DCB-Eq), freshwater eutrophication (FE, kg P-Eq), human toxicity (HT, kg 1,4-DCB-Eq), ionizing radiation (IR, kg U235-Eq), marine ecotoxicity (MEC, kg 1,4-DCB-Eq), marine eutrophication (ME, kg N-Eq), mineral resource depletion (MD, kg Fe-Eq), natural land transformation (NLT, m²), ozone depletion (OD, kg CFC-11-Eq), particulate matter formation (PMF, kg PM10-Eq), photochemical oxidant formation (POF, kg NMVOC), terrestrial acidification (TA, $\mathrm{kg} \mathrm{SO}_{2}$-Eq), terrestrial ecotoxicity (TEC, kg 1,4-DCB-Eq), urban land occupation (ULO, $\mathrm{m}^{2} \mathrm{a}$ ), water depletion (WD, $\mathrm{m}^{3}$ ). 


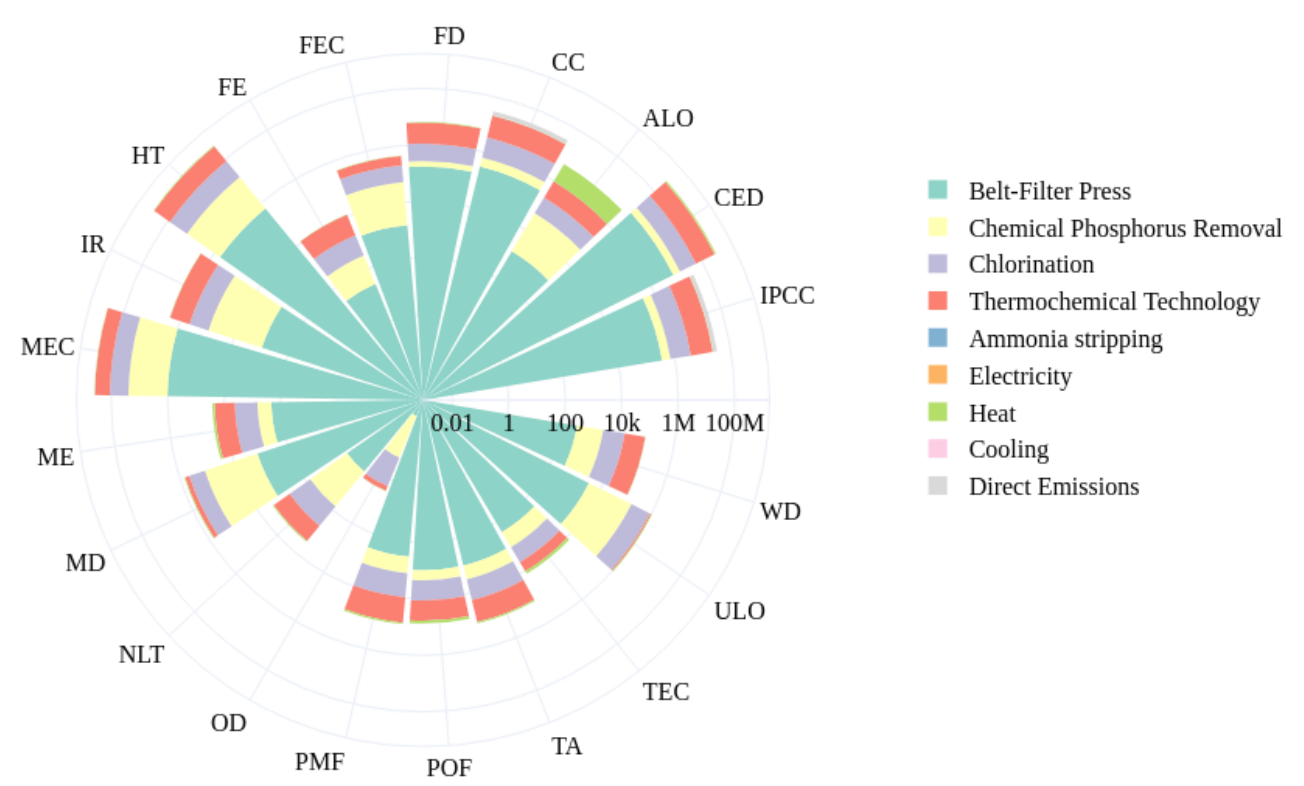

Figure S3. Environmental profile of the retrofitting design with slow pyrolysis on a logarithmic scale. The radial axis indicates the absolute quantity of each midpoint impact category, where " $\mathrm{k}$ " refers to one thousand and "M" stands for one million. Acronyms go counter clockwise: global warming potential (GWP) according to IPCC (IPCC, $\mathrm{kg} \mathrm{CO}_{2}$-Eq), cumulative energy demand (CED, MJ-Eq), agricultural land occupation (ALO, m²a), climate change (CC, $\mathrm{kg} \mathrm{CO}_{2}$ $\mathrm{Eq}$ ), fossil fuel depletion (FD, kg oil-Eq), freshwater ecotoxicity (FEC, kg 1,4-DCB-Eq), freshwater eutrophication (FE, kg P-Eq), human toxicity (HT, kg 1,4-DCB-Eq), ionizing radiation (IR, kg U235-Eq), marine ecotoxicity (MEC, kg 1,4-DCB-Eq), marine eutrophication (ME, kg N$\mathrm{Eq}$ ), mineral resource depletion (MD, kg Fe-Eq), natural land transformation (NLT, m²), ozone depletion (OD, kg CFC-11-Eq), particulate matter formation (PMF, kg PM10-Eq), photochemical oxidant formation (POF, kg NMVOC), terrestrial acidification (TA, $\mathrm{kg} \mathrm{SO}$-Eq), terrestrial ecotoxicity (TEC, kg 1,4-DCB-Eq), urban land occupation (ULO, $\mathrm{m}^{2} \mathrm{a}$ ), water depletion (WD, $\mathrm{m}^{3}$ ). 


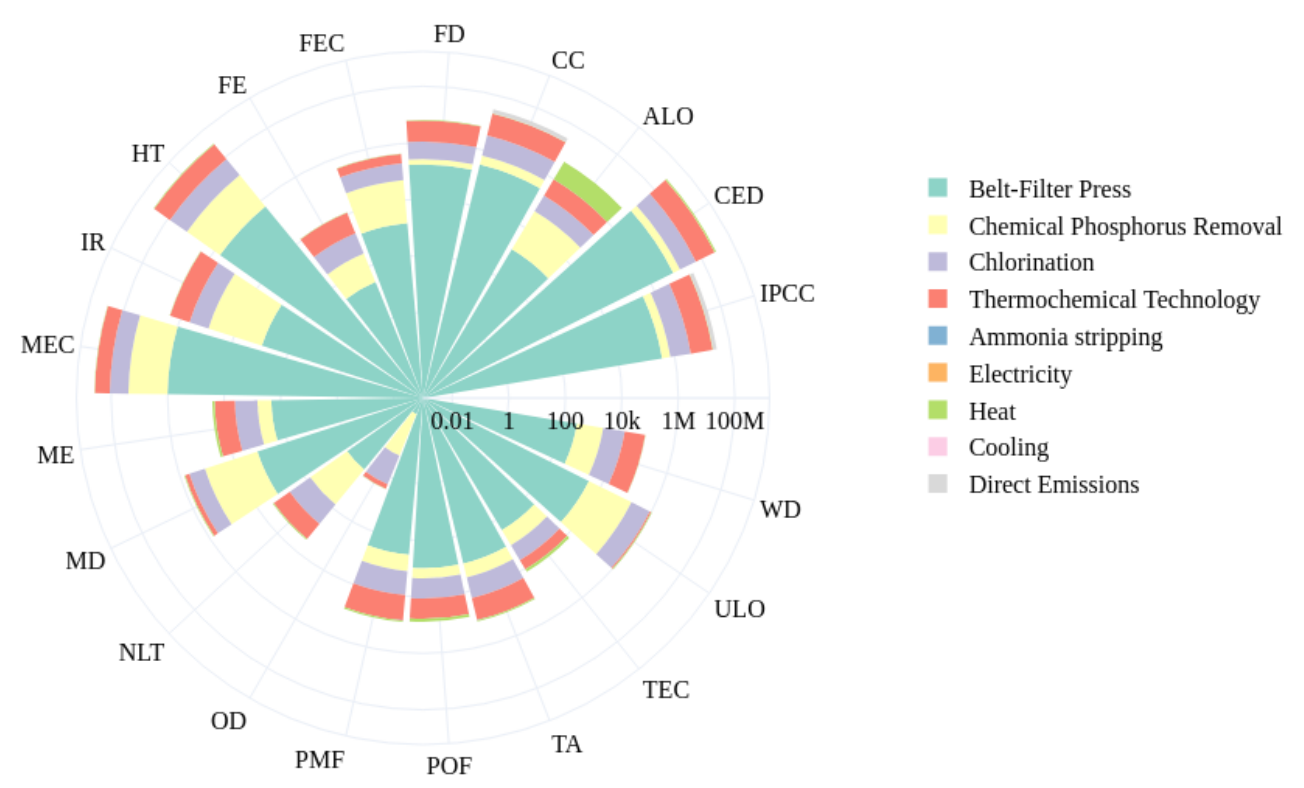

Figure S4. Environmental profile of the retrofitting design with fast pyrolysis on a logarithmic scale. The radial axis indicates the absolute quantity of each midpoint impact category, where " $\mathrm{k}$ " refers to one thousand and "M" stands for one million. Acronyms go counter clockwise: global warming potential (GWP) according to IPCC (IPCC, $\mathrm{kg} \mathrm{CO}_{2}$-Eq), cumulative energy demand (CED, MJ-Eq), agricultural land occupation (ALO, $\mathrm{m}^{2} \mathrm{a}$ ), climate change (CC, $\mathrm{kg} \mathrm{CO}_{2}$-Eq), fossil fuel depletion (FD, kg oil-Eq), freshwater ecotoxicity (FEC, kg 1,4-DCB-Eq), freshwater eutrophication (FE, kg P-Eq), human toxicity (HT, kg 1,4-DCB-Eq), ionizing radiation (IR, kg U235-Eq), marine ecotoxicity (MEC, kg 1,4-DCB-Eq), marine eutrophication (ME, kg N-Eq), mineral resource depletion (MD, kg Fe-Eq), natural land transformation (NLT, m²), ozone depletion (OD, kg CFC-11-Eq), particulate matter formation (PMF, kg PM10-Eq), photochemical oxidant formation (POF, kg NMVOC), terrestrial acidification (TA, $\mathrm{kg} \mathrm{SO}_{2}$-Eq), terrestrial ecotoxicity (TEC, kg 1,4-DCB-Eq), urban land occupation (ULO, $\mathrm{m}^{2} \mathrm{a}$ ), water depletion (WD, $\mathrm{m}^{3}$ ). 


\section{S2. Key input parameters used in CapdetWorks and capital cost estimates for the reference design}

Table S1. Summary of Air Supply System for the reference design.

\begin{tabular}{|l|l|l|}
\hline Description & Value & Units \\
\hline Blower System for Entire Plant & \multicolumn{2}{l|}{} \\
\hline Design Information & 22600 & scfm \\
\hline Minimum air flow capacity & 1.5 & \\
\hline Safety factor & 33900 & scfm \\
\hline Requested air flow capacity & 33900 & scfm \\
\hline Total capacity of blowers & 2 & \\
\hline Number of blowers in use & 3 & \\
\hline Total number of blowers & 17000 & scfm \\
\hline Capacity of individual blowers & 579000 & $\$$ \\
\hline Estimated cost of an installed blower & 1850 & sqft \\
\hline Blower building area & 2150000 & $\$$ \\
\hline Costs & 1740000 & $\$$ \\
\hline Construction and equipment cost & 203000 & $\$$ \\
\hline Installed Blower Cost & 214000 & $\$$ \\
\hline Building Cost & & \\
\hline Misc Costs & & \\
\hline
\end{tabular}

Table S2. Summary of Chemical Feed System for Iron for the reference design.

\begin{tabular}{|l|l|l|}
\hline Description & Value & Units \\
\hline Iron Salt Solution Feed System & 6360 & $\mathrm{lb} / \mathrm{d}$ \\
\hline Quantities & 2190 & $\mathrm{lb} / \mathrm{d}$ \\
\hline Ferric chloride dosage rate & 1810 & gpd(US) \\
\hline Iron salt dosage rate as equivalent iron & 2030 & pers-hrs/yr \\
\hline Liquid chemical solution fed & & \\
\hline Operation labor required &
\end{tabular}


Table S3. Summary of Influent Pump Station for the reference design.

\begin{tabular}{|c|c|c|}
\hline Description & Value & Units \\
\hline \multicolumn{3}{|l|}{ Pump Station } \\
\hline \multicolumn{3}{|l|}{ Design Information } \\
\hline Volume of wet well & 19100 & cuft \\
\hline Width of wet well & 135 & $\mathrm{ft}$ \\
\hline Depth of the pumping station & 29.4 & $\mathrm{ft}$ \\
\hline Length of the pumping station & 32.2 & $\mathrm{ft}$ \\
\hline Width of the pumping station & 168 & $\mathrm{ft}$ \\
\hline Minimum depth of water in wet well & 8.43 & $\mathrm{ft}$ \\
\hline Area of pump building & 1100 & sqft \\
\hline Peak capacity of pumps & 39.9 & MGD(US) \\
\hline Firm pumping capacity & 39.9 & MGD(US) \\
\hline Total dynamic head - average conditions & 44 & $\mathrm{ft}$ \\
\hline \multicolumn{3}{|l|}{ Quantities } \\
\hline Operation labor required & 986 & pers-hrs/yr \\
\hline Maintenance labor required & 926 & pers-hrs/yr \\
\hline Electrical energy required & 807000 & $\mathrm{kWh} / \mathrm{yr}$ \\
\hline Volume of earthwork required & 612000 & cuft \\
\hline Volume of slab concrete required & 55800 & cuft \\
\hline Volume of wall concrete required & 16800 & cuft \\
\hline Capacity per pump & 13800 & gpm(US) \\
\hline Number of constant speed pumps & 3 & \\
\hline Number of variable speed pumps & 0 & \\
\hline Diameter of discharge header pipe & 37.6 & in \\
\hline Total dynamic head & 53.5 & $\mathrm{ft}$ \\
\hline Size of selected pump & 24 & in \\
\hline
\end{tabular}




\begin{tabular}{|l|l|l|}
\hline Specific speed of pump & 3570 & \\
\hline Pump rotating speed & 672 & $\mathrm{rpm}$ \\
\hline Motor size required & 194 & $\mathrm{HP}$ \\
\hline Size of selected motor & 200 & $\mathrm{HP}$ \\
\hline Width of pump system & 5.4 & $\mathrm{ft}$ \\
\hline Length of pump system & 23.5 & $\mathrm{ft}$ \\
\hline Length of the dry well & 32.2 & $\mathrm{ft}$ \\
\hline Width of the dry well & 32.5 & $\mathrm{ft}$ \\
\hline Costs & & \\
\hline Construction and equipment cost & 2730000 & $\$$ \\
\hline Earthwork Cost & 181000 & $\$$ \\
\hline Wall Concrete Cost & 405000 & $\$$ \\
\hline Slab Concrete Cost & 724000 & $\$$ \\
\hline Building Cost & 121000 & $\$$ \\
\hline Installed Pump Equipment Cost & 880000 & $\$$ \\
\hline Misc Costs & 416000 & $\$$ \\
\hline
\end{tabular}

Table S4. Summary of Preliminary Treatment for the reference design.

\begin{tabular}{|l|l|l|}
\hline Description & Value & Units \\
\hline Preliminary Treatment & \multicolumn{2}{l|}{} \\
\hline Design Information & 0.25 & \multicolumn{2}{l|}{} \\
\hline Mechanically Cleaned Bar Screen & 1.5 & in \\
\hline Bar size & 30 & in \\
\hline Bar spacing & 0.0206 & degrees \\
\hline Slope of bars from horizontal & 2.5 & $\mathrm{ft}$ \\
\hline Head loss through screen & 2.5 & $\mathrm{ft} / \mathrm{s}$ \\
\hline Approach velocity & 3 & $\mathrm{ft} / \mathrm{s}$ \\
\hline Average flow through velocity (screen) & $\mathrm{ft} / \mathrm{s}$ \\
\hline Maximum flow through velocity (screen) & &
\end{tabular}




\begin{tabular}{|c|c|c|}
\hline Screen channel width & 8.07 & $\mathrm{ft}$ \\
\hline Average channel depth & 1 & $\mathrm{ft}$ \\
\hline \multicolumn{3}{|l|}{ Horizontal Flow Grit Chamber } \\
\hline Maximum flow & 46.2 & cuft/s \\
\hline Average flow & 20.2 & cuft/s \\
\hline Minimum flow & 15.4 & cuft/s \\
\hline Temperature & 10 & $\operatorname{deg} \mathrm{C}$ \\
\hline Maximum flow through velocity (grit chamber) & 1.5 & $\mathrm{ft} / \mathrm{s}$ \\
\hline Average flow through velocity (grit chamber) & 1 & $\mathrm{ft} / \mathrm{s}$ \\
\hline Size of smallest particle $100 \%$ removed & 0.2 & $\mathrm{~mm}$ \\
\hline Specific gravity of particle & 2.65 & \\
\hline Number of units & 2 & \\
\hline Maximum flow/unit & 23.1 & cuft/s \\
\hline Width of channel & 3.85 & $\mathrm{ft}$ \\
\hline Depth of channel & 4 & $\mathrm{ft}$ \\
\hline Length of channel & 144 & $\mathrm{ft}$ \\
\hline Settling velocity of particle & 0.0707 & $\mathrm{ft} / \mathrm{s}$ \\
\hline Slope of channel bottom & 0.00048 & \\
\hline Allowance for currents & 1.7 & \\
\hline Manning coefficient & 0.035 & \\
\hline Hydraulic retention time & 1.6 & $\min$ \\
\hline Volume of grit & 52.4 & cuft/d \\
\hline \multicolumn{3}{|l|}{ Costs } \\
\hline Construction and equipment cost & 1390000 & $\$$ \\
\hline
\end{tabular}

Table S5. Summary of Primary Clarification for the reference design.

\begin{tabular}{|l|l|l|}
\hline Description & Value & Units \\
\hline Primary Clarification & \\
\hline Design Information & \\
\hline
\end{tabular}




\begin{tabular}{|c|c|c|}
\hline Surface area & 30200 & sqft \\
\hline Surface area per circular clarifier & 7560 & sqft \\
\hline Diameter of each circular clarifier & 99 & $\mathrm{ft}$ \\
\hline Number of clarifiers per battery & 4 & \\
\hline Number of batteries & 1 & \\
\hline Solids loading rate & 1.03 & $\mathrm{lb} /(\mathrm{sqft} \cdot \mathrm{d})$ \\
\hline Hydraulic retention time & 3.66 & $\mathrm{hr}$ \\
\hline Weir length & 2020 & $\mathrm{ft}$ \\
\hline Volume of sludge generated & 54200 & gpd(US) \\
\hline \multicolumn{3}{|l|}{ Quantities } \\
\hline Operation labor required & 1990 & pers-hrs/yr \\
\hline Maintenance labor required & 1110 & pers-hrs/yr \\
\hline Electrical energy required & 15500 & $\mathrm{kWh} / \mathrm{yr}$ \\
\hline Volume of earthwork required & 413000 & cuft \\
\hline Slab thickness & 10.1 & in \\
\hline Volume of slab concrete required & 29600 & cuft \\
\hline Wall thickness & 11.5 & in \\
\hline Volume of wall concrete required & 13000 & cuft \\
\hline \multicolumn{3}{|l|}{ Costs } \\
\hline Construction and equipment cost & 1700000 & $\$$ \\
\hline Earthwork Cost & 122000 & $\$$ \\
\hline Wall Concrete Cost & 313000 & $\$$ \\
\hline Slab Concrete Cost & 384000 & $\$$ \\
\hline Installed Equipment Cost & 624000 & $\$$ \\
\hline Misc Costs & 260000 & $\$$ \\
\hline \multicolumn{3}{|l|}{ Waste Sludge Pumping } \\
\hline \multicolumn{3}{|l|}{\begin{tabular}{|l|} 
Design Information \\
\end{tabular}} \\
\hline Average daily pumping rate & 0.0542 & MGD(US) \\
\hline Total pumping capacity & 0.0542 & MGD(US) \\
\hline
\end{tabular}




\begin{tabular}{|l|l|l|}
\hline Design capacity per pump & 18.8 & gpm(US) \\
\hline Number of pumps & 3 & \\
\hline Number of batteries & 1 & \\
\hline Firm pumping capacity & 0.0542 & MGD(US) \\
\hline Quantities & \multicolumn{2}{|l|}{} \\
\hline Operation labor required & 303 & pers-hrs/yr \\
\hline Maintenance labor required & 234 & pers-hrs/yr \\
\hline Electrical energy required & 1830 & kWh/yr \\
\hline Volume of earthwork required & 1610 & cuft \\
\hline Area of pump building & 201 & sqft \\
\hline Costs & \multicolumn{2}{|l|}{} \\
\hline Construction and equipment cost & 43700 & $\$$ \\
\hline Earthwork Cost & 477 & $\$$ \\
\hline Pump Building Cost & 22100 & $\$$ \\
\hline Installed Pump Cost & 14400 & $\$$ \\
\hline Misc Costs & 6660 & $\$$ \\
\hline
\end{tabular}

Table S6. Summary of Anaerobic Digestion for the reference design.

\begin{tabular}{|l|l|l|}
\hline Description & Value & Units \\
\hline Anaerobic Digestion & \multicolumn{2}{l|}{} \\
\hline Design Information & 50 & $\%$ \\
\hline Percent VSS destroyed & 5 & $\%$ \\
\hline Solids concentration in digester & 28 & $\mathrm{~d}$ \\
\hline Detention time & 28 & $\mathrm{ft}$ \\
\hline Digester depth & 70 & $\mathrm{ft}$ \\
\hline Digester diameter & 475000 & $\mathrm{cuft}$ \\
\hline Effective digester volume & 4 & \\
\hline Number of digesters per battery & 3 & \\
\hline Number of primary digesters per battery & & \\
\hline
\end{tabular}




\begin{tabular}{|c|c|c|}
\hline Number of secondary digesters per battery & 1 & \\
\hline Number of batteries & 1 & \\
\hline Gas produced & 111 & cuft/min \\
\hline Heat required & 3260000 & BTU/hr \\
\hline Digester gas required & 126 & cuft/min \\
\hline Total natural gas required & 1590000 & cuft/yr \\
\hline \multicolumn{3}{|l|}{ Quantities } \\
\hline Operation labor required & 3190 & pers-hrs/yr \\
\hline Maintenance labor required & 2050 & pers-hrs/yr \\
\hline Electrical energy required & 311000 & $\mathrm{kWh} / \mathrm{yr}$ \\
\hline Volume of earthwork required & 474000 & cuft \\
\hline Slab thickness & 11 & in \\
\hline Volume of slab concrete required & 15400 & cuft \\
\hline Wall thickness & 21.5 & in \\
\hline Volume of wall concrete required & 53700 & cuft \\
\hline Sidewater depth & 28 & $\mathrm{ft}$ \\
\hline Surface area/floor of 2-story control bldg & 2390 & sqft \\
\hline Piping size & 8 & in \\
\hline Length of total piping system & 1320 & $\mathrm{ft}$ \\
\hline Number of 90 degree elbows & 52 & \\
\hline Number of tees & 102 & \\
\hline Number of plug valves & 74 & \\
\hline Total dry solids treated & 18.1 & ton(short)/d \\
\hline \multicolumn{3}{|l|}{ Costs } \\
\hline Construction and equipment cost & 8450000 & $\$$ \\
\hline Earthwork Cost & 140000 & $\$$ \\
\hline Wall Concrete Cost & 1290000 & $\$$ \\
\hline Slab Concrete Cost & 200000 & $\$$ \\
\hline Building Cost & 263000 & $\$$ \\
\hline
\end{tabular}




\begin{tabular}{|l|l|l|}
\hline Piping System Cost & 804000 & $\$$ \\
\hline Floating Cover Cost & 2950000 & $\$$ \\
\hline Gas Recirculation Units Cost & 888000 & $\$$ \\
\hline Heating Units Cost & 670000 & $\$$ \\
\hline Gas Safety Equipment Cost & 254000 & $\$$ \\
\hline Installed Pumps Cost & 158000 & $\$$ \\
\hline
\end{tabular}

Table S7. Summary of Belt-Filter Press for the reference design.

\begin{tabular}{|l|l|l|}
\hline Description & Value & Units \\
\hline Belt-Filter Press & \multicolumn{2}{l|}{} \\
\hline Design Information & 2 & m \\
\hline Belt filter width & 2 & \\
\hline Number of units & \multicolumn{2}{l|}{} \\
\hline Hydraulic loading per unit per meter of belt width & 70 & gpm(US) \\
\hline Hydraulic loading required per meter of belt width & 170 & gpm(US) \\
\hline Final solids content & 20 & $\%$ \\
\hline Solids capture fraction & 0.998 & \\
\hline Quantities & & \\
\hline Operation labor required & 812 & pers-hrs/yr \\
\hline Maintenance labor required & 203 & pers-hrs/yr \\
\hline Power & 222000 & $\mathrm{kWh} / \mathrm{yr}$ \\
\hline Polymer required & 107000 & $\mathrm{lb} / \mathrm{yr}$ \\
\hline Dry solids produced & 29200 & $\mathrm{lb} / \mathrm{d}$ \\
\hline Belt filter(s) & 636000 & $\$$ \\
\hline Building & 358000 & $\$$ \\
\hline Installation & 159000 & $\$$ \\
\hline Polymer system & 235000 & $\$$ \\
\hline Feed pumps & 70000 & $\$$ \\
\hline Conveyor system & 159000 & $\$$ \\
\hline
\end{tabular}




\begin{tabular}{|l|l|l|}
\hline \multicolumn{2}{|l|}{ Costs } & 1620000 \\
\hline Construction and equipment cost & $\$ 38000$ & $\$$ \\
\hline Building Cost & 235000 & $\$$ \\
\hline Polymer System Cost & 70000 & $\$$ \\
\hline Feed Pumps Cost & 159000 & $\$$ \\
\hline Conveyor System Cost & 795000 & $\$$ \\
\hline Installed Belt Filter & & \\
\hline
\end{tabular}

Table S8. Summary of Plug Flow Activated Sludge for the reference design.

\begin{tabular}{|c|c|c|}
\hline Description & Value & Units \\
\hline \multicolumn{3}{|l|}{ Plug-Flow Aerated Bioreactor } \\
\hline \multicolumn{3}{|l|}{ Design Information } \\
\hline \multicolumn{3}{|l|}{ Carbon \& Nitrification Design } \\
\hline Design SRT for design at winter temperature & 15 & $\mathrm{~d}$ \\
\hline Design SS & 2500 & $\mathrm{mg} / \mathrm{L}$ \\
\hline Calculated VSS & 1690 & $\mathrm{mg} / \mathrm{L}$ \\
\hline Calculated VSS:TSS ratio & 0.678 & mg VSS/mg SS \\
\hline Total volume of reactors & 41000 & $\mathrm{~m} 3$ \\
\hline Length of parallel train & 103 & $\mathrm{~m}$ \\
\hline Width of parallel train & 10 & $\mathrm{~m}$ \\
\hline Sidewater depth & 5 & $\mathrm{~m}$ \\
\hline Number of batteries & 1 & \\
\hline Number of parallel trains per battery & 8 & \\
\hline Number of cells within one train & 3 & \\
\hline Total number of dividing walls between zones & 16 & \\
\hline Hydraulic retention time & 19.6 & $\mathrm{hr}$ \\
\hline F/M ratio & 0.114 & kg BOD/kg MLSS/d \\
\hline Volumetric BOD loading & 0.193 & $\mathrm{~kg} \mathrm{BOD} / \mathrm{m} 3 / \mathrm{d}$ \\
\hline Observed yield (VSS basis) & 0.755 & g VSS/g BOD \\
\hline
\end{tabular}




\begin{tabular}{|c|c|c|}
\hline Observed yield (TSS basis) & 0.742 & g TSS/g BOD \\
\hline Amount of alkalinity required & 221 & $\mathrm{gCaCO} / \mathrm{m} 3$ \\
\hline Amount of sludge generated & 6840 & $\mathrm{~kg} / \mathrm{d}$ \\
\hline Sludge recycle rate & 16800 & $\mathrm{~m} 3 / \mathrm{d}$ \\
\hline Nitrogen requirement for biomass growth & 5.74 & $\mathrm{mg} / \mathrm{L}$ \\
\hline Phosphorus requirement for biomass growth & 1.15 & $\mathrm{mg} / \mathrm{L}$ \\
\hline Oxygen requirement to meet average demand & 16500 & $\mathrm{~kg} / \mathrm{d}$ \\
\hline Air flow required to meet average demand & 38300 & $\mathrm{~N} \mathrm{~m} 3 / \mathrm{hr}$ \\
\hline Design air flow & 15.5 & $\mathrm{~N} \mathrm{~m} 3 / \mathrm{min} / 1000 \mathrm{~m} 3$ \\
\hline \multicolumn{3}{|l|}{ Quantities } \\
\hline Operation labor required & 4150 & pers-hrs/yr \\
\hline Maintenance labor required & 2570 & pers-hrs/yr \\
\hline Electrical energy required & 5820000 & $\mathrm{kWh} / \mathrm{yr}$ \\
\hline Volume of earthwork required & 664000 & cuft \\
\hline Volume of slab concrete required & 206000 & cuft \\
\hline Volume of wall concrete required & 122000 & cuft \\
\hline Handrail length & 4400 & $\mathrm{ft}$ \\
\hline Number of diffusers per train & 1410 & \\
\hline Fine bubble diffuser floor coverage & 5.63 & $\%$ \\
\hline Number of swing arm headers per train & 14 & \\
\hline \multicolumn{3}{|l|}{ Costs } \\
\hline Construction and equipment cost & 11600000 & $\$$ \\
\hline Earthwork Cost & 197000 & $\$$ \\
\hline Wall Concrete Cost & 2940000 & $\$$ \\
\hline Slab Concrete Cost & 2680000 & $\$$ \\
\hline Handrail Cost & 330000 & $\$$ \\
\hline Installed Aerator Equipment Cost & 3130000 & $\$$ \\
\hline Air Piping Cost & 1190000 & $\$$ \\
\hline Misc Costs & 1150000 & $\$$ \\
\hline
\end{tabular}




\begin{tabular}{|c|c|c|}
\hline \multicolumn{3}{|l|}{ Sludge Recycle Pumping } \\
\hline \multicolumn{3}{|l|}{ Design Information } \\
\hline Average daily pumping rate & 4.43 & MGD(US) \\
\hline Total pumping capacity & 8.86 & MGD(US) \\
\hline Design capacity per pump & 3070 & gpm(US) \\
\hline Number of pumps & 3 & \\
\hline Number of batteries & 1 & \\
\hline Firm pumping capacity & 4.43 & MGD(US) \\
\hline \multicolumn{3}{|l|}{ Quantities } \\
\hline Operation labor required & 533 & pers-hrs/yr \\
\hline Maintenance labor required & 449 & pers-hrs/yr \\
\hline Electrical energy required & 148000 & $\mathrm{kWh} / \mathrm{yr}$ \\
\hline Volume of earthwork required & 3000 & cuft \\
\hline Area of pump building & 375 & sqft \\
\hline \multicolumn{3}{|l|}{ Costs } \\
\hline Construction and equipment cost & 210000 & $\$$ \\
\hline Earthwork Cost & 888 & $\$$ \\
\hline Pump Building Cost & 41200 & $\$$ \\
\hline Installed Pump Cost & 136000 & $\$$ \\
\hline Misc Costs & 32000 & $\$$ \\
\hline
\end{tabular}

Table S9. Summary of Gravity Thickening for the reference design.

\begin{tabular}{|l|l|l|}
\hline Description & Value & Units \\
\hline Gravity Thickening & \multicolumn{2}{l|}{} \\
\hline Design Information & 1.91 & $\%$ \\
\hline Initial concentration & 5 & $\%$ \\
\hline Thickened concentration & 10 & $\mathrm{lb} /(\mathrm{sqft} \cdot \mathrm{d})$ \\
\hline Mass loading & 62.7 & $\mathrm{gal}(\mathrm{US}) /(\mathrm{sqft} \cdot \mathrm{d})$ \\
\hline Hydraulic loading & &
\end{tabular}




\begin{tabular}{|l|l|l|}
\hline Hydraulic retention time & 25.8 & $\mathrm{hr}$ \\
\hline Number of tanks & 2 & \\
\hline Tank volume & 36200 & cuft \\
\hline Depth & 9 & $\mathrm{ft}$ \\
\hline Surface area per tank & 2010 & sqft \\
\hline Tank diameter & 51 & $\mathrm{ft}$ \\
\hline Quantities & \multicolumn{2}{|l|}{} \\
\hline Amount of sludge generated & 20.1 & ton(short)/d \\
\hline Volume of thickened sludge & 82700 & gpd(US) \\
\hline Operation labor required & 940 & pers-hrs/yr \\
\hline Maintenance labor required & 624 & pers-hrs/yr \\
\hline Electrical energy required & 11100 & kWh/yr \\
\hline Volume of earthwork required & 49700 & cuft \\
\hline Slab thickness & 10.1 & in \\
\hline Volume of slab concrete required & 4220 & cuft \\
\hline Wall thickness & 11.5 & in \\
\hline Volume of wall concrete required & 3440 & cuft \\
\hline Costs & \multicolumn{2}{|l|}{} \\
\hline Construction and equipment cost & 477000 & $\$$ \\
\hline Earthwork Cost & 14700 & $\$$ \\
\hline Wall Concrete Cost & 82900 & $\$$ \\
\hline Slab Concrete Cost & 54700 & $\$$ \\
\hline Installed Equipment Cost & 252000 & $\$$ \\
\hline Misc Costs & 72800 & $\$$ \\
\hline
\end{tabular}

Table S10. Summary of Hauling and Land Filling for the reference design.

\begin{tabular}{|l|l|l|}
\hline Description & Value & Units \\
\hline Sludge Hauling and Land Filling & \\
\hline Design Information & \\
\hline
\end{tabular}




\begin{tabular}{|c|c|c|}
\hline Volume of sludge hauled & 72.2 & cuyd/d \\
\hline Truck capacity & 22 & cuyd \\
\hline Round trip time to disposal site & 1 & $\mathrm{hr}$ \\
\hline Truck loading time & 0.75 & $\mathrm{hr}$ \\
\hline Operational hours per day & 8 & $\mathrm{hr}$ \\
\hline Number of trucks required & 1 & \\
\hline Distance to disposal site & 10 & miles \\
\hline \multicolumn{3}{|l|}{ Quantities } \\
\hline Total sludge volume hauled & 72.2 & cuyd/d \\
\hline Maximum anticipated landfill downtime & 30 & $\mathrm{~d}$ \\
\hline Anticipated sludge storage height & 8 & $\mathrm{ft}$ \\
\hline Sludge storage shed area & 7310 & sqft \\
\hline Width of sludge storage shed slab & 60.5 & $\mathrm{ft}$ \\
\hline Length of sludge storage shed slab & 121 & $\mathrm{ft}$ \\
\hline Volume of earthwork required & 19400 & cuft \\
\hline Volume of slab concrete required & 8140 & cuft \\
\hline Surface area of canopy roof & 7310 & sqft \\
\hline Round trip haul distance & 20 & miles \\
\hline Round trips per day per truck & 4 & \\
\hline Distance traveled per year per truck & 20000 & miles \\
\hline Sludge hauled & 63.9 & ton(short)/d \\
\hline Operation labor required & 1130 & pers-hrs/yr \\
\hline LandFilling cost & 36900 & $\$ / y r$ \\
\hline \multicolumn{3}{|l|}{ Costs } \\
\hline Construction and equipment cost & 623000 & $\$$ \\
\hline Earthwork Cost & 5760 & $\$$ \\
\hline Slab Concrete Cost & 106000 & $\$$ \\
\hline Canopy Roof Cost & 146000 & $\$$ \\
\hline Vehicle Cost & 365000 & $\$$ \\
\hline
\end{tabular}


Table S11. Summary of Secondary Clarifier for the reference design.

\begin{tabular}{|c|c|c|}
\hline Description & Value & Units \\
\hline \multicolumn{3}{|l|}{ Secondary Clarification } \\
\hline \multicolumn{3}{|l|}{ Design Information } \\
\hline Surface area & 60400 & sqft \\
\hline Surface area per circular clarifier & 15100 & sqft \\
\hline Diameter of each circular clarifier & 139 & $\mathrm{ft}$ \\
\hline Number of clarifiers per battery & 4 & \\
\hline Number of batteries & 1 & \\
\hline Solids loading rate & 6.12 & $\mathrm{lb} /(\mathrm{sqft} \cdot \mathrm{d})$ \\
\hline Hydraulic retention time & 7.34 & $\mathrm{hr}$ \\
\hline Designed surface overflow rate & 500 & $\operatorname{gal}(\mathrm{US}) /(\mathrm{sqft} \cdot \mathrm{d})$ \\
\hline Weir length & 2010 & $\mathrm{ft}$ \\
\hline Volume of wasted sludge & 176000 & gpd(US) \\
\hline \multicolumn{3}{|l|}{ Quantities } \\
\hline Operation labor required & 3020 & pers-hrs/yr \\
\hline Maintenance labor required & 1690 & pers-hrs/yr \\
\hline Electrical energy required & 23200 & $\mathrm{kWh} / \mathrm{yr}$ \\
\hline Volume of earthwork required & 917000 & cuft \\
\hline Slab thickness & 10.1 & in \\
\hline Volume of slab concrete required & 57100 & cuft \\
\hline Wall thickness & 11.5 & in \\
\hline Volume of wall concrete required & 18000 & cuft \\
\hline \multicolumn{3}{|l|}{ Costs } \\
\hline Construction and equipment cost & 2690000 & $\$$ \\
\hline Earthwork Cost & 272000 & $\$$ \\
\hline Wall Concrete Cost & 434000 & $\$$ \\
\hline Slab Concrete Cost & 740000 & $\$$ \\
\hline Installed Equipment Cost & 837000 & $\$$ \\
\hline
\end{tabular}




\begin{tabular}{|c|c|c|}
\hline Misc Costs & 411000 & $\$$ \\
\hline \multicolumn{3}{|l|}{ Waste Sludge Pumping } \\
\hline \multicolumn{3}{|l|}{ Design Information } \\
\hline Average daily pumping rate & 0.176 & MGD(US) \\
\hline Total pumping capacity & 0.176 & MGD(US) \\
\hline Design capacity per pump & 61 & gpm(US) \\
\hline Number of pumps & 3 & \\
\hline Number of batteries & 1 & \\
\hline Firm pumping capacity & 0.176 & MGD(US) \\
\hline \multicolumn{3}{|l|}{ Quantities } \\
\hline Operation labor required & 352 & pers-hrs/yr \\
\hline Maintenance labor required & 278 & pers-hrs/yr \\
\hline Electrical energy required & 5910 & $\mathrm{kWh} / \mathrm{yr}$ \\
\hline Volume of earthwork required & 1630 & cuft \\
\hline Area of pump building & 203 & sqft \\
\hline \multicolumn{3}{|l|}{ Costs } \\
\hline Construction and equipment cost & 55500 & $\$$ \\
\hline Earthwork Cost & 482 & $\$$ \\
\hline Pump Building Cost & 22400 & $\$$ \\
\hline Installed Pump Cost & 24200 & $\$$ \\
\hline Misc Costs & 8470 & $\$$ \\
\hline
\end{tabular}

Table S12. Summary of Chemical Phosphorus Removal for the reference design.

\begin{tabular}{|l|l|l|}
\hline Description & Value & Units \\
\hline Chemical Phosphorus Removal & \multicolumn{2}{|l|}{} \\
\hline Design Information & Equivalent Iron & \\
\hline Chemical used & 20 & $\mathrm{~g} / \mathrm{m} 3$ \\
\hline Chemical dosage & 363000 & $\mathrm{~kg} / \mathrm{yr}$ \\
\hline Mass of chemical per year & &
\end{tabular}




\begin{tabular}{|l|l|l|}
\hline Chemical sludge production & 61.2 & $\mathrm{~g} / \mathrm{m} 3$ \\
\hline Organic sludge production & 0.659 & $\mathrm{~g} / \mathrm{m} 3$ \\
\hline
\end{tabular}

Table S13. Summary of Tertiary Clarification for the reference design.

\begin{tabular}{|c|c|c|}
\hline Description & Value & Units \\
\hline \multicolumn{3}{|l|}{ Primary Clarification } \\
\hline \multicolumn{3}{|l|}{ Design Information } \\
\hline Surface area & 30000 & sqft \\
\hline Surface area per circular clarifier & 7500 & sqft \\
\hline Diameter of each circular clarifier & 98 & $\mathrm{ft}$ \\
\hline Number of clarifiers per battery & 4 & \\
\hline Number of batteries & 1 & \\
\hline Solids loading rate & 0.262 & lb/(sqft·d) \\
\hline Hydraulic retention time & 3.7 & $\mathrm{hr}$ \\
\hline Weir length & 2000 & $\mathrm{ft}$ \\
\hline Volume of sludge generated & 22500 & gpd(US) \\
\hline \multicolumn{3}{|l|}{ Quantities } \\
\hline Operation labor required & 1980 & pers-hrs/yr \\
\hline Maintenance labor required & 1100 & pers-hrs/yr \\
\hline Electrical energy required & 15500 & $\mathrm{kWh} / \mathrm{yr}$ \\
\hline Volume of earthwork required & 403000 & cuft \\
\hline Slab thickness & 10.1 & in \\
\hline Volume of slab concrete required & 29000 & cuft \\
\hline Wall thickness & 11.5 & in \\
\hline Volume of wall concrete required & 12900 & cuft \\
\hline \multicolumn{3}{|l|}{ Costs } \\
\hline Construction and equipment cost & 1680000 & $\$$ \\
\hline Earthwork Cost & 120000 & $\$$ \\
\hline Wall Concrete Cost & 310000 & $\$$ \\
\hline
\end{tabular}




\begin{tabular}{|c|c|c|}
\hline Slab Concrete Cost & 376000 & $\$$ \\
\hline Installed Equipment Cost & 619000 & $\$$ \\
\hline Misc Costs & 256000 & $\$$ \\
\hline \multicolumn{3}{|l|}{ Waste Sludge Pumping } \\
\hline \multicolumn{3}{|l|}{ Design Information } \\
\hline Average daily pumping rate & 0.0225 & MGD(US) \\
\hline Total pumping capacity & 0.0225 & MGD(US) \\
\hline Design capacity per pump & 7.8 & gpm(US) \\
\hline Number of pumps & 3 & \\
\hline Number of batteries & 1 & \\
\hline Firm pumping capacity & 0.0225 & MGD(US) \\
\hline \multicolumn{3}{|l|}{ Quantities } \\
\hline Operation labor required & 270 & pers-hrs/yr \\
\hline Maintenance labor required & 205 & pers-hrs/yr \\
\hline Electrical energy required & 759 & $\mathrm{kWh} / \mathrm{yr}$ \\
\hline Volume of earthwork required & 1600 & cuft \\
\hline Area of pump building & 200 & sqft \\
\hline \multicolumn{3}{|l|}{ Costs } \\
\hline Construction and equipment cost & 38100 & $\$$ \\
\hline Earthwork Cost & 475 & $\$$ \\
\hline Pump Building Cost & 22000 & $\$$ \\
\hline Installed Pump Cost & 9770 & $\$$ \\
\hline Misc Costs & 5810 & $\$$ \\
\hline
\end{tabular}

Table S14. Summary of Chlorination for the reference design.

\begin{tabular}{|l|l|l|}
\hline Description & Value & Units \\
\hline Chlorination & \multicolumn{2}{l|}{} \\
\hline Design Information & 625000 & gal(US) \\
\hline Volume of tank & &
\end{tabular}




\begin{tabular}{|c|c|c|}
\hline Average chlorine required & 1090 & $\mathrm{lb} / \mathrm{d}$ \\
\hline Peak chlorine required & 2500 & $\mathrm{lb} / \mathrm{d}$ \\
\hline Influent coliform count & 10000000 & $/ 100 \mathrm{ml}$ \\
\hline Effluent coliform count & 29.2 & $/ 100 \mathrm{ml}$ \\
\hline \multicolumn{3}{|l|}{ Quantities } \\
\hline Operational labor required & 1670 & pers-hrs/yr \\
\hline Maintenance labor required & 471 & pers-hrs/yr \\
\hline Electrical energy required & 138000 & $\mathrm{kWh} / \mathrm{yr}$ \\
\hline Volume of earthwork required & 35500 & cuft \\
\hline Volume of slab concrete required & 8370 & cuft \\
\hline Volume of wall concrete required & 11300 & cuft \\
\hline Number of chlorinators and evaporators & 1 & \\
\hline Chlorination building area & 220 & sqft \\
\hline Number of chlorine cylinders & 17 & \\
\hline Area of chlorine storage building & 2380 & sqft \\
\hline \multicolumn{3}{|l|}{ Costs } \\
\hline Construction and equipment cost & 1040000 & $\$$ \\
\hline Earthwork Cost & 10500 & $\$$ \\
\hline Wall Concrete Cost & 272000 & $\$$ \\
\hline Slab Concrete Cost & 109000 & $\$$ \\
\hline Installed Equipment Cost & 422000 & $\$$ \\
\hline Building Cost & 24200 & $\$$ \\
\hline Storage Building Cost & 131000 & $\$$ \\
\hline Misc Costs & 70400 & $\$$ \\
\hline
\end{tabular}

\section{S3. Key input parameters used in CapdetWorks and capital cost estimates for the retrofitting design with hydrothermal liquefaction}

Table S15. Summary of Air Supply System for the retrofitting design with hydrothermal liquefaction. 


\begin{tabular}{|l|l|l|}
\hline Blower System for Entire Plant & scfm \\
\hline Design Information & 22000 & \\
\hline Minimum air flow capacity & 1.5 & scfm \\
\hline Safety factor & 33000 & scfm \\
\hline Requested air flow capacity & 33000 & \\
\hline Total capacity of blowers & 2 & \\
\hline Number of blowers in use & 3 & scfm \\
\hline Total number of blowers & 16500 & $\$$ \\
\hline Capacity of individual blowers & 570000 & sqft \\
\hline Estimated cost of an installed blower & 1840 & \multicolumn{2}{|l|}{} \\
\hline Blower building area & \multicolumn{2}{|l|}{} \\
\hline Costs & 2120000 & $\$$ \\
\hline Construction and equipment cost &
\end{tabular}

Table S16. Summary of Chemical Feed System for Iron for the retrofitting design with hydrothermal liquefaction.

\begin{tabular}{|l|l|l|}
\hline Description & Value & Units \\
\hline Iron Salt Solution Feed System & \multicolumn{2}{l|}{} \\
\hline Quantities & 6360 & $\mathrm{lb} / \mathrm{d}$ \\
\hline Ferric chloride dosage rate & 2190 & $\mathrm{lb} / \mathrm{d}$ \\
\hline Iron salt dosage rate as equivalent iron & 1810 & gpd(US) \\
\hline Liquid chemical solution fed & 2030 & pers-hrs/yr \\
\hline Operation labor required & \multicolumn{2}{|l}{} \\
\hline Costs & 342000 & $\$$ \\
\hline Construction and equipment cost & \multicolumn{2}{|l|}{} \\
\hline
\end{tabular}

Table S17. Summary of Chemical Feed System for Lime for the retrofitting design with hydrothermal liquefaction.

\begin{tabular}{|l|l|l|}
\hline Description & Value & Units \\
\hline Lime Solution Feed System & \\
\hline Quantities & \\
\hline
\end{tabular}




\begin{tabular}{|l|l|l|}
\hline Lime feed rate of $\mathrm{Ca}(\mathrm{OH}) 2$ & 73.3 & lb/d \\
\hline Liquid chemical solution fed & 147 & gpd(US) \\
\hline O\&M labor required & 680 & pers-hrs/yr \\
\hline Dry material handling and mixing labor required & 379 & pers-hrs/yr \\
\hline Total operation labor required & 1060 & pers-hrs/yr \\
\hline Costs & 109000 & $\$$ \\
\hline Construction and equipment cost & \multicolumn{2}{|l}{} \\
\hline
\end{tabular}

Table S18. Summary of Influent Pump Station for the retrofitting design with hydrothermal liquefaction.

\begin{tabular}{|l|l|l|}
\hline Description & Value & Units \\
\hline \multicolumn{2}{|l|}{ Pump Station } & \multicolumn{2}{l|}{} \\
\hline Design Information & 19100 & $\mathrm{cuft}$ \\
\hline Volume of wet well & 135 & $\mathrm{ft}$ \\
\hline Width of wet well & 29.4 & $\mathrm{ft}$ \\
\hline Depth of the pumping station & 32.2 & $\mathrm{ft}$ \\
\hline Length of the pumping station & 168 & $\mathrm{ft}$ \\
\hline Width of the pumping station & 8.43 & $\mathrm{ft}$ \\
\hline Minimum depth of water in wet well & 1100 & sqft \\
\hline Area of pump building & 39.9 & $\mathrm{MGD(US)}$ \\
\hline Peak capacity of pumps & 39.9 & $\mathrm{MGD(US)}$ \\
\hline Firm pumping capacity & 44 & $\mathrm{ft}$ \\
\hline Total dynamic head - average conditions & & \\
\hline Quantities & 986 & $\mathrm{pers}-\mathrm{hrs} / \mathrm{yr}$ \\
\hline Operation labor required & 926 & $\mathrm{pers}-\mathrm{hrs} / \mathrm{yr}$ \\
\hline Maintenance labor required & 807000 & $\mathrm{kWh} / \mathrm{yr}$ \\
\hline Electrical energy required & 612000 & $\mathrm{cuft}$ \\
\hline Volume of earthwork required & 55800 & cuft \\
\hline Volume of slab concrete required & 16800 & $\mathrm{cuft}$ \\
\hline Volume of wall concrete required & 13800 & $\mathrm{gpm}(\mathrm{US})$ \\
\hline Capacity per pump & \multicolumn{2}{|l|}{} \\
\hline
\end{tabular}




\begin{tabular}{|l|l|l|}
\hline Number of constant speed pumps & 3 & \\
\hline Number of variable speed pumps & 0 & \\
\hline Diameter of discharge header pipe & 37.6 & in \\
\hline Total dynamic head & 53.5 & $\mathrm{ft}$ \\
\hline Size of selected pump & 24 & $\mathrm{in}$ \\
\hline Specific speed of pump & 3570 & \\
\hline Pump rotating speed & 672 & $\mathrm{rpm}$ \\
\hline Motor size required & 194 & $\mathrm{HP}$ \\
\hline Size of selected motor & 200 & $\mathrm{HP}$ \\
\hline Width of pump system & 5.4 & $\mathrm{ft}$ \\
\hline Length of pump system & 23.5 & $\mathrm{ft}$ \\
\hline Length of the dry well & 32.2 & $\mathrm{ft}$ \\
\hline Width of the dry well & 32.5 & $\mathrm{ft}$ \\
\hline Costs & & \\
\hline Construction and equipment cost & 2730000 & $\$$ \\
\hline Earthwork Cost & 181000 & $\$$ \\
\hline Wall Concrete Cost & 405000 & $\$$ \\
\hline Slab Concrete Cost & 724000 & $\$$ \\
\hline Building Cost & 121000 & $\$$ \\
\hline Installed Pump Equipment Cost & 880000 & $\$$ \\
\hline Misc Costs & 416000 & $\$$ \\
\hline
\end{tabular}

Table S19. Summary of Preliminary Treatment for the retrofitting design with hydrothermal liquefaction.

\begin{tabular}{|l|l|l|}
\hline Description & Value & Units \\
\hline Preliminary Treatment & \multicolumn{2}{l|}{} \\
\hline Design Information & \multicolumn{2}{l|}{} \\
\hline Mechanically Cleaned Bar Screen & 0.25 & in \\
\hline Bar size & 1.5 & in \\
\hline Bar spacing & 30 & degrees \\
\hline Slope of bars from horizontal &
\end{tabular}




\begin{tabular}{|c|c|c|}
\hline Head loss through screen & 0.0206 & $\mathrm{ft}$ \\
\hline Approach velocity & 2.5 & $\mathrm{ft} / \mathrm{s}$ \\
\hline Average flow through velocity (screen) & 2.5 & $\mathrm{ft} / \mathrm{s}$ \\
\hline Maximum flow through velocity (screen) & 3 & $\mathrm{ft} / \mathrm{s}$ \\
\hline Screen channel width & 8.07 & $\mathrm{ft}$ \\
\hline Average channel depth & 1 & $\mathrm{ft}$ \\
\hline \multicolumn{3}{|l|}{ Horizontal Flow Grit Chamber } \\
\hline Maximum flow & 46.2 & $\mathrm{cuft} / \mathrm{s}$ \\
\hline Average flow & 20.2 & $\mathrm{cuft} / \mathrm{s}$ \\
\hline Minimum flow & 15.4 & $\mathrm{cuft} / \mathrm{s}$ \\
\hline Temperature & 10 & $\operatorname{deg} C$ \\
\hline Maximum flow through velocity (grit chamber) & 1.5 & $\mathrm{ft} / \mathrm{s}$ \\
\hline Average flow through velocity (grit chamber) & 1 & $\mathrm{ft} / \mathrm{s}$ \\
\hline Size of smallest particle $100 \%$ removed & 0.2 & $\mathrm{~mm}$ \\
\hline Specific gravity of particle & 2.65 & \\
\hline Number of units & 2 & \\
\hline Maximum flow/unit & 23.1 & cuft/s \\
\hline Width of channel & 3.85 & $\mathrm{ft}$ \\
\hline Depth of channel & 4 & $\mathrm{ft}$ \\
\hline Length of channel & 144 & $\mathrm{ft}$ \\
\hline Settling velocity of particle & 0.0707 & $\mathrm{ft} / \mathrm{s}$ \\
\hline Slope of channel bottom & 0.00048 & \\
\hline Allowance for currents & 1.7 & \\
\hline Manning coefficient & 0.035 & \\
\hline Hydraulic retention time & 1.6 & $\min$ \\
\hline Volume of grit & 52.4 & cuft/d \\
\hline \multicolumn{3}{|l|}{ Costs } \\
\hline Construction and equipment cost & 1390000 & $\$$ \\
\hline
\end{tabular}

Table S20. Summary of Counter Current Ammonia Stripping for the retrofitting design with hydrothermal liquefaction. 


\begin{tabular}{|l|l|l|}
\hline Description & Value & Units \\
\hline Counter Current Ammonia Stripping & \multicolumn{2}{l|}{} \\
\hline Design Information & \multicolumn{2}{l|}{} \\
\hline Liquid loading rate & 600 & $\mathrm{lb} /(\mathrm{sqft} \cdot \mathrm{hr})$ \\
\hline Gas loading rate & 1800 & $\mathrm{lb} /(\mathrm{sqft} \cdot \mathrm{hr})$ \\
\hline Tower surface area & 25.5 & $\mathrm{sqft}$ \\
\hline Tower height & 166 & $\mathrm{ft}$ \\
\hline Initial ammonia concentration & 650 & $\mathrm{mg} / \mathrm{L}$ \\
\hline Effluent ammonia concentration & 2 & $\mathrm{mg} / \mathrm{L}$ \\
\hline Waste water flow & 0.044 & $\mathrm{MGD}(\mathrm{US})$ \\
\hline Lime required & 73.3 & $\mathrm{lb} / \mathrm{d}$ \\
\hline Costs & \multicolumn{2}{|l}{} \\
\hline Construction and equipment cost & 40500 & $\$$ \\
\hline
\end{tabular}

Table S21. Summary of Primary Clarification for the retrofitting design with hydrothermal liquefaction.

\begin{tabular}{|l|l|l|}
\hline Description & Value & Units \\
\hline Primary Clarification & \multicolumn{2}{l|}{} \\
\hline Design Information & 30200 & sqft \\
\hline Surface area & 7560 & sqft \\
\hline Surface area per circular clarifier & 99 & $\mathrm{ft}$ \\
\hline Diameter of each circular clarifier & 4 & \\
\hline Number of clarifiers per battery & 1 & \\
\hline Number of batteries & 1.03 & $\mathrm{lb} /(\mathrm{sqft} \cdot \mathrm{d})$ \\
\hline Solids loading rate & 3.66 & $\mathrm{hr}$ \\
\hline Hydraulic retention time & 2020 & $\mathrm{ft}$ \\
\hline Weir length & 54100 & $\mathrm{gpd}(\mathrm{US})$ \\
\hline Volume of sludge generated & & \\
\hline Quantities & 1990 & $\mathrm{pers}-\mathrm{hrs} / \mathrm{yr}$ \\
\hline Operation labor required & 1110 & $\mathrm{pers}-\mathrm{hrs} / \mathrm{yr}$ \\
\hline Maintenance labor required & &
\end{tabular}




\begin{tabular}{|c|c|c|}
\hline Electrical energy required & 15500 & $\mathrm{kWh} / \mathrm{yr}$ \\
\hline Volume of earthwork required & 413000 & cuft \\
\hline Slab thickness & 10.1 & in \\
\hline Volume of slab concrete required & 29600 & cuft \\
\hline Wall thickness & 11.5 & in \\
\hline Volume of wall concrete required & 13000 & cuft \\
\hline \multicolumn{3}{|l|}{ Costs } \\
\hline Construction and equipment cost & 1700000 & $\$$ \\
\hline Earthwork Cost & 122000 & $\$$ \\
\hline Wall Concrete Cost & 313000 & $\$$ \\
\hline Slab Concrete Cost & 384000 & $\$$ \\
\hline Installed Equipment Cost & 624000 & $\$$ \\
\hline Misc Costs & 260000 & $\$$ \\
\hline \multicolumn{3}{|l|}{ Waste Sludge Pumping } \\
\hline \multicolumn{3}{|l|}{\begin{tabular}{|l|} 
Design Information \\
\end{tabular}} \\
\hline Average daily pumping rate & 0.0542 & MGD(US) \\
\hline Total pumping capacity & 0.0542 & MGD(US) \\
\hline Design capacity per pump & 18.8 & gpm(US) \\
\hline Number of pumps & 3 & \\
\hline Number of batteries & 1 & \\
\hline Firm pumping capacity & 0.0542 & MGD(US) \\
\hline \multicolumn{3}{|l|}{ Quantities } \\
\hline Operation labor required & 303 & pers-hrs/yr \\
\hline Maintenance labor required & 234 & pers-hrs/yr \\
\hline Electrical energy required & 1830 & $\mathrm{kWh} / \mathrm{yr}$ \\
\hline Volume of earthwork required & 1610 & cuft \\
\hline Area of pump building & 201 & sqft \\
\hline \multicolumn{3}{|l|}{ Costs } \\
\hline Construction and equipment cost & 43700 & $\$$ \\
\hline Earthwork Cost & 477 & $\$$ \\
\hline
\end{tabular}




\begin{tabular}{|l|l|l|}
\hline Pump Building Cost & 22100 & $\$$ \\
\hline Installed Pump Cost & 14400 & $\$$ \\
\hline Misc Costs & 6660 & $\$$ \\
\hline
\end{tabular}

Table S22. Summary of Anaerobic Digestion for the retrofitting design with hydrothermal liquefaction.

\begin{tabular}{|c|c|c|}
\hline Description & Value & Units \\
\hline \multicolumn{3}{|l|}{ Anaerobic Digestion } \\
\hline \multicolumn{3}{|l|}{ Design Information } \\
\hline Percent VSS destroyed & 50 & $\%$ \\
\hline Solids concentration in digester & 5 & $\%$ \\
\hline Detention time & 28 & d \\
\hline Digester depth & 28 & $\mathrm{ft}$ \\
\hline Digester diameter & 70 & $\mathrm{ft}$ \\
\hline Effective digester volume & 475000 & cuft \\
\hline Number of digesters per battery & 4 & \\
\hline Number of primary digesters per battery & 3 & \\
\hline Number of secondary digesters per battery & 1 & \\
\hline Number of batteries & 1 & \\
\hline Gas produced & 111 & cuft/min \\
\hline Heat required & 3260000 & BTU/hr \\
\hline Digester gas required & 126 & cuft/min \\
\hline Total natural gas required & 1600000 & cuft/yr \\
\hline \multicolumn{3}{|l|}{ Quantities } \\
\hline Operation labor required & 3200 & pers-hrs/yr \\
\hline Maintenance labor required & 2050 & pers-hrs/yr \\
\hline Electrical energy required & 312000 & $\mathrm{kWh} / \mathrm{yr}$ \\
\hline Volume of earthwork required & 474000 & cuft \\
\hline Slab thickness & 11 & in \\
\hline Volume of slab concrete required & 15400 & cuft \\
\hline Wall thickness & 21.5 & in \\
\hline
\end{tabular}




\begin{tabular}{|l|l|l|}
\hline Volume of wall concrete required & 53700 & cuft \\
\hline Sidewater depth & 28 & $\mathrm{ft}$ \\
\hline Surface area/floor of 2-story control bldg & 2390 & $\mathrm{sqft}$ \\
\hline Piping size & 8 & in \\
\hline Length of total piping system & 1320 & $\mathrm{ft}$ \\
\hline Number of 90 degree elbows & 52 & \\
\hline Number of tees & 102 & \\
\hline Number of plug valves & 74 & \\
\hline Total dry solids treated & 18.1 & ton(short)/d \\
\hline Costs & & \\
\hline Construction and equipment cost & 8450000 & $\$$ \\
\hline Earthwork Cost & 140000 & $\$$ \\
\hline Wall Concrete Cost & 1290000 & $\$$ \\
\hline Slab Concrete Cost & 200000 & $\$$ \\
\hline Building Cost & 263000 & $\$$ \\
\hline Piping System Cost & 804000 & $\$$ \\
\hline Floating Cover Cost & 2950000 & $\$$ \\
\hline Gas Recirculation Units Cost & 888000 & $\$$ \\
\hline Heating Units Cost & 670000 & $\$$ \\
\hline Gas Safety Equipment Cost & 254000 & $\$$ \\
\hline Installed Pumps Cost & 158000 & $\$$ \\
\hline
\end{tabular}

Table S23. Summary of Plug Flow Activated Sludge for the retrofitting design with hydrothermal liquefaction.

\begin{tabular}{|l|l|l|}
\hline Description & Value & Units \\
\hline Plug-Flow Aerated Bioreactor & & \\
\hline Design Information & \multicolumn{2}{l|}{} \\
\hline Carbon Only Design & 15 & $\mathrm{~d}$ \\
\hline Design SRT for design at winter temperature & 2500 & $\mathrm{mg} / \mathrm{L}$ \\
\hline Design SS & 1690 & $\mathrm{mg} / \mathrm{L}$ \\
\hline Calculated VSS & &
\end{tabular}




\begin{tabular}{|c|c|c|}
\hline Calculated VSS:TSS ratio & 0.677 & mg VSS/mg SS \\
\hline Total volume of reactors & 41000 & $\mathrm{~m} 3$ \\
\hline Length of parallel train & 103 & $\mathrm{~m}$ \\
\hline Width of parallel train & 10 & $\mathrm{~m}$ \\
\hline Sidewater depth & 5 & $\mathrm{~m}$ \\
\hline Number of batteries & 1 & \\
\hline Number of parallel trains per battery & 8 & \\
\hline Number of cells within one train & 3 & \\
\hline Total number of dividing walls between zones & 16 & \\
\hline Hydraulic retention time & 19.6 & $\mathrm{hr}$ \\
\hline $\mathrm{F} / \mathrm{M}$ ratio & 0.114 & kg BOD/kg MLSS/d \\
\hline Volumetric BOD loading & 0.193 & $\mathrm{~kg} \mathrm{BOD} / \mathrm{m} 3 / \mathrm{d}$ \\
\hline Observed yield (VSS basis) & 0.754 & g VSS/g BOD \\
\hline Observed yield (TSS basis) & 0.742 & g TSS/g BOD \\
\hline Amount of alkalinity required & 206 & $\mathrm{gCaCO} / \mathrm{m} 3$ \\
\hline Amount of sludge generated & 6830 & $\mathrm{~kg} / \mathrm{d}$ \\
\hline Sludge recycle rate & 16800 & $\mathrm{~m} 3 / \mathrm{d}$ \\
\hline Nitrogen requirement for biomass growth & 5.72 & $\mathrm{mg} / \mathrm{L}$ \\
\hline Phosphorus requirement for biomass growth & 1.14 & $\mathrm{mg} / \mathrm{L}$ \\
\hline Oxygen requirement to meet average demand & 16000 & $\mathrm{~kg} / \mathrm{d}$ \\
\hline Air flow required to meet average demand & 37300 & $\mathrm{~N} \mathrm{~m} 3 / \mathrm{hr}$ \\
\hline Design air flow & 15.1 & $\mathrm{~N} \mathrm{~m} 3 / \mathrm{min} / 1000 \mathrm{~m} 3$ \\
\hline \multicolumn{3}{|l|}{ Quantities } \\
\hline Operation labor required & 4090 & pers-hrs/yr \\
\hline Maintenance labor required & 2530 & pers-hrs/yr \\
\hline Electrical energy required & 5670000 & $\mathrm{kWh} / \mathrm{yr}$ \\
\hline Volume of earthwork required & 664000 & cuft \\
\hline Volume of slab concrete required & 206000 & cuft \\
\hline Volume of wall concrete required & 122000 & cuft \\
\hline Handrail length & 4400 & $\mathrm{ft}$ \\
\hline
\end{tabular}




\begin{tabular}{|c|c|c|}
\hline Number of diffusers per train & 1380 & \\
\hline Fine bubble diffuser floor coverage & 5.49 & $\%$ \\
\hline Number of swing arm headers per train & 14 & \\
\hline \multicolumn{3}{|l|}{ Costs } \\
\hline Construction and equipment cost & 11600000 & $\$$ \\
\hline Earthwork Cost & 197000 & $\$$ \\
\hline Wall Concrete Cost & 2940000 & $\$$ \\
\hline Slab Concrete Cost & 2680000 & $\$$ \\
\hline Handrail Cost & 330000 & $\$$ \\
\hline Installed Aerator Equipment Cost & 3110000 & $\$$ \\
\hline Air Piping Cost & 1170000 & $\$$ \\
\hline Misc Costs & 1150000 & $\$$ \\
\hline \multicolumn{3}{|l|}{ Sludge Recycle Pumping } \\
\hline \multicolumn{3}{|l|}{ Design Information } \\
\hline Average daily pumping rate & 4.43 & MGD(US) \\
\hline Total pumping capacity & 8.85 & MGD(US) \\
\hline Design capacity per pump & 3070 & gpm(US) \\
\hline Number of pumps & 3 & \\
\hline Number of batteries & 1 & \\
\hline Firm pumping capacity & 4.43 & MGD(US) \\
\hline \multicolumn{3}{|l|}{ Quantities } \\
\hline Operation labor required & 533 & pers-hrs/yr \\
\hline Maintenance labor required & 449 & pers-hrs/yr \\
\hline Electrical energy required & 148000 & $\mathrm{kWh} / \mathrm{yr}$ \\
\hline Volume of earthwork required & 3000 & cuft \\
\hline Area of pump building & 375 & sqft \\
\hline \multicolumn{3}{|l|}{ Costs } \\
\hline Construction and equipment cost & 210000 & $\$$ \\
\hline
\end{tabular}

Table S24. Summary of Gravity Thickening for the retrofitting design with hydrothermal liquefaction. 


\begin{tabular}{|c|c|c|}
\hline Description & Value & Units \\
\hline \multicolumn{3}{|l|}{ Gravity Thickening } \\
\hline \multicolumn{3}{|l|}{ Design Information } \\
\hline Initial concentration & 1.91 & $\%$ \\
\hline Thickened concentration & 5 & $\%$ \\
\hline Mass loading & 10 & $\mathrm{lb} /(\mathrm{sqft} \cdot \mathrm{d})$ \\
\hline Hydraulic loading & 62.6 & $\operatorname{gal}(\mathrm{US}) /(\mathrm{sqft} \cdot \mathrm{d})$ \\
\hline Hydraulic retention time & 25.8 & $\mathrm{hr}$ \\
\hline Number of tanks & 2 & \\
\hline Tank volume & 36300 & cuft \\
\hline Depth & 9 & $\mathrm{ft}$ \\
\hline Surface area per tank & 2010 & sqft \\
\hline Tank diameter & 51 & $\mathrm{ft}$ \\
\hline \multicolumn{3}{|l|}{ Quantities } \\
\hline Amount of sludge generated & 20.1 & ton(short)/d \\
\hline Volume of thickened sludge & 82800 & gpd(US) \\
\hline Operation labor required & 941 & pers-hrs/yr \\
\hline Maintenance labor required & 625 & pers-hrs/yr \\
\hline Electrical energy required & 11100 & $\mathrm{kWh} / \mathrm{yr}$ \\
\hline Volume of earthwork required & 49700 & cuft \\
\hline Slab thickness & 10.1 & in \\
\hline Volume of slab concrete required & 4220 & cuft \\
\hline Wall thickness & 11.5 & in \\
\hline Volume of wall concrete required & 3440 & cuft \\
\hline \multicolumn{3}{|l|}{ Costs } \\
\hline Construction and equipment cost & 477000 & $\$$ \\
\hline Earthwork Cost & 14700 & $\$$ \\
\hline Wall Concrete Cost & 82900 & $\$$ \\
\hline Slab Concrete Cost & 54700 & $\$$ \\
\hline Installed Equipment Cost & 252000 & $\$$ \\
\hline
\end{tabular}




\begin{tabular}{|l|l|l|}
\hline Misc Costs & 72800 & $\$$ \\
\hline
\end{tabular}

Table S25. Summary of Secondary Clarifier for the retrofitting design with hydrothermal liquefaction.

\begin{tabular}{|c|c|c|}
\hline Description & Value & Units \\
\hline \multicolumn{3}{|l|}{ Secondary Clarification } \\
\hline \multicolumn{3}{|l|}{ Design Information } \\
\hline Surface area & 60400 & sqft \\
\hline Surface area per circular clarifier & 15100 & sqft \\
\hline Diameter of each circular clarifier & 139 & $\mathrm{ft}$ \\
\hline Number of clarifiers per battery & 4 & \\
\hline Number of batteries & 1 & \\
\hline Solids loading rate & 6.12 & $\mathrm{lb} /(\mathrm{sqft} \cdot \mathrm{d})$ \\
\hline Hydraulic retention time & 7.34 & $\mathrm{hr}$ \\
\hline Designed surface overflow rate & 500 & gal(US)/(sqft·d) \\
\hline Weir length & 2010 & $\mathrm{ft}$ \\
\hline Volume of wasted sludge & 175000 & gpd(US) \\
\hline \multicolumn{3}{|l|}{ Quantities } \\
\hline Operation labor required & 3020 & pers-hrs/yr \\
\hline Maintenance labor required & 1690 & pers-hrs/yr \\
\hline Electrical energy required & 23200 & $\mathrm{kWh} / \mathrm{yr}$ \\
\hline Volume of earthwork required & 917000 & cuft \\
\hline Slab thickness & 10.1 & in \\
\hline Volume of slab concrete required & 57100 & cuft \\
\hline Wall thickness & 11.5 & in \\
\hline Volume of wall concrete required & 18000 & cuft \\
\hline \multicolumn{3}{|l|}{ Costs } \\
\hline Construction and equipment cost & 2690000 & $\$$ \\
\hline Earthwork Cost & 272000 & $\$$ \\
\hline Wall Concrete Cost & 434000 & $\$$ \\
\hline Slab Concrete Cost & 740000 & $\$$ \\
\hline
\end{tabular}




\begin{tabular}{|c|c|c|}
\hline Installed Equipment Cost & 837000 & $\$$ \\
\hline Misc Costs & 411000 & $\$$ \\
\hline \multicolumn{3}{|l|}{ Waste Sludge Pumping } \\
\hline \multicolumn{3}{|l|}{ Design Information } \\
\hline Average daily pumping rate & 0.175 & MGD(US) \\
\hline Total pumping capacity & 0.175 & MGD(US) \\
\hline Design capacity per pump & 60.9 & gpm(US) \\
\hline Number of pumps & 3 & \\
\hline Number of batteries & 1 & \\
\hline Firm pumping capacity & 0.175 & MGD(US) \\
\hline \multicolumn{3}{|l|}{ Quantities } \\
\hline Operation labor required & 352 & pers-hrs/yr \\
\hline Maintenance labor required & 278 & pers-hrs/yr \\
\hline Electrical energy required & 5900 & $\mathrm{kWh} / \mathrm{yr}$ \\
\hline Volume of earthwork required & 1630 & cuft \\
\hline Area of pump building & 203 & sqft \\
\hline \multicolumn{3}{|l|}{ Costs } \\
\hline Construction and equipment cost & 55500 & $\$$ \\
\hline Earthwork Cost & 482 & $\$$ \\
\hline Pump Building Cost & 22400 & $\$$ \\
\hline Installed Pump Cost & 24200 & $\$$ \\
\hline Misc Costs & 8460 & $\$$ \\
\hline
\end{tabular}

Table S26. Summary of Chemical Phosphorus Removal for the retrofitting design with hydrothermal liquefaction.

\begin{tabular}{|l|l|l|}
\hline Description & Value & Units \\
\hline Chemical Phosphorus Removal & \multicolumn{2}{|l|}{} \\
\hline Design Information & Equivalent Iron & \\
\hline Chemical used & 20 & $\mathrm{~g} / \mathrm{m} 3$ \\
\hline Chemical dosage & 363000 & $\mathrm{~kg} / \mathrm{yr}$ \\
\hline Mass of chemical per year &
\end{tabular}




\begin{tabular}{|l|l|l|}
\hline Chemical sludge production & 61.2 & $\mathrm{~g} / \mathrm{m} 3$ \\
\hline Organic sludge production & 0.659 & $\mathrm{~g} / \mathrm{m} 3$ \\
\hline
\end{tabular}

Table S27. Summary of Tertiary Clarification for the retrofitting design with hydrothermal liquefaction.

\begin{tabular}{|c|c|c|}
\hline Description & Value & Units \\
\hline \multicolumn{3}{|l|}{ Primary Clarification } \\
\hline \multicolumn{3}{|l|}{ Design Information } \\
\hline Surface area & 30000 & sqft \\
\hline Surface area per circular clarifier & 7500 & sqft \\
\hline Diameter of each circular clarifier & 98 & $\mathrm{ft}$ \\
\hline Number of clarifiers per battery & 4 & \\
\hline Number of batteries & 1 & \\
\hline Solids loading rate & 0.262 & $\mathrm{lb} /(\mathrm{sqft} \cdot \mathrm{d})$ \\
\hline Hydraulic retention time & 3.7 & $\mathrm{hr}$ \\
\hline Weir length & 2000 & $\mathrm{ft}$ \\
\hline Volume of sludge generated & 22700 & $\operatorname{gpd}(\mathrm{US})$ \\
\hline \multicolumn{3}{|l|}{ Quantities } \\
\hline Operation labor required & 1980 & pers-hrs/yr \\
\hline Maintenance labor required & 1100 & pers-hrs/yr \\
\hline Electrical energy required & 15500 & $\mathrm{kWh} / \mathrm{yr}$ \\
\hline Volume of earthwork required & 403000 & cuft \\
\hline Slab thickness & 10.1 & in \\
\hline Volume of slab concrete required & 29000 & cuft \\
\hline Wall thickness & 11.5 & in \\
\hline Volume of wall concrete required & 12900 & cuft \\
\hline \multicolumn{3}{|l|}{ Costs } \\
\hline Construction and equipment cost & 1680000 & $\$$ \\
\hline Earthwork Cost & 120000 & $\$$ \\
\hline Wall Concrete Cost & 310000 & $\$$ \\
\hline Slab Concrete Cost & 376000 & $\$$ \\
\hline
\end{tabular}




\begin{tabular}{|c|c|c|}
\hline Installed Equipment Cost & 619000 & $\$$ \\
\hline Misc Costs & 256000 & $\$$ \\
\hline \multicolumn{3}{|l|}{ Waste Sludge Pumping } \\
\hline \multicolumn{3}{|l|}{ Design Information } \\
\hline Average daily pumping rate & 0.0227 & MGD(US) \\
\hline Total pumping capacity & 0.0227 & MGD(US) \\
\hline Design capacity per pump & 7.88 & gpm(US) \\
\hline Number of pumps & 3 & \\
\hline Number of batteries & 1 & \\
\hline Firm pumping capacity & 0.0227 & MGD(US) \\
\hline \multicolumn{3}{|l|}{ Quantities } \\
\hline Operation labor required & 271 & pers-hrs/yr \\
\hline Maintenance labor required & 206 & pers-hrs/yr \\
\hline Electrical energy required & 768 & $\mathrm{kWh} / \mathrm{yr}$ \\
\hline Volume of earthwork required & 1600 & cuft \\
\hline Area of pump building & 200 & sqft \\
\hline \multicolumn{3}{|l|}{ Costs } \\
\hline Construction and equipment cost & 38200 & $\$$ \\
\hline Earthwork Cost & 475 & $\$$ \\
\hline Pump Building Cost & 22000 & $\$$ \\
\hline Installed Pump Cost & 9820 & $\$$ \\
\hline Misc Costs & 5820 & $\$$ \\
\hline
\end{tabular}

Table S28. Summary of Chlorination for the retrofitting design with hydrothermal liquefaction.

\begin{tabular}{|l|l|l|}
\hline Description & Value & Units \\
\hline Chlorination & \multicolumn{2}{l|}{} \\
\hline Design Information & 625000 & gal(US) \\
\hline Volume of tank & 1090 & $\mathrm{lb} / \mathrm{d}$ \\
\hline Average chlorine required & 2500 & $\mathrm{lb} / \mathrm{d}$ \\
\hline Peak chlorine required & & \\
\hline
\end{tabular}




\begin{tabular}{|l|l|l|}
\hline Influent coliform count & 10000000 & $/ 100 \mathrm{ml}$ \\
\hline Effluent coliform count & 29.2 & $/ 100 \mathrm{ml}$ \\
\hline Quantities & \multicolumn{2}{l|}{} \\
\hline Operational labor required & 1670 & pers-hrs/yr \\
\hline Maintenance labor required & 471 & pers-hrs/yr \\
\hline Electrical energy required & 138000 & $\mathrm{kWh} / \mathrm{yr}$ \\
\hline Volume of earthwork required & 35500 & cuft \\
\hline Volume of slab concrete required & 8370 & cuft \\
\hline Volume of wall concrete required & 11300 & cuft \\
\hline Number of chlorinators and evaporators & 1 & \\
\hline Chlorination building area & 220 & sqft \\
\hline Number of chlorine cylinders & 17 & \\
\hline Area of chlorine storage building & 2380 & sqft \\
\hline Costs & & \multicolumn{2}{|l|}{} \\
\hline Construction and equipment cost & 1040000 & $\$$ \\
\hline Earthwork Cost & 10500 & $\$$ \\
\hline Wall Concrete Cost & 272000 & $\$$ \\
\hline Slab Concrete Cost & 109000 & $\$$ \\
\hline Installed Equipment Cost & 422000 & $\$$ \\
\hline Building Cost & 24200 & $\$$ \\
\hline Storage Building Cost & 131000 & $\$$ \\
\hline Misc Costs & 70400 & \multicolumn{2}{|l|}{} \\
\hline
\end{tabular}

\section{S4. Key input parameters used in CapdetWorks and capital cost estimates for the retrofitting design with pyrolysis}

Table S29. Summary of Air Supply System for the retrofitting design with pyrolysis.

\begin{tabular}{|l|l|l|}
\hline Description & Value & Units \\
\hline Blower System for Entire Plant & \multicolumn{2}{|l|}{} \\
\hline Design Information & 20300 & scfm \\
\hline Minimum air flow capacity &
\end{tabular}




\begin{tabular}{|l|l|l|}
\hline Safety factor & 1.5 & \\
\hline Requested air flow capacity & 30500 & scfm \\
\hline Total capacity of blowers & 30500 & scfm \\
\hline Number of blowers in use & 2 & \\
\hline Total number of blowers & 3 & \\
\hline Capacity of individual blowers & 15200 & scfm \\
\hline Estimated cost of an installed blower & 544000 & $\$$ \\
\hline Blower building area & 1800 & sqft \\
\hline Costs & & \\
\hline Construction and equipment cost & 2030000 & $\$$ \\
\hline Installed Blower Cost & 1630000 & $\$$ \\
\hline Building Cost & 198000 & $\$$ \\
\hline Misc Costs & 201000 & $\$$ \\
\hline
\end{tabular}

Table S30. Summary of Chemical Feed System for Lime for the retrofitting design with pyrolysis.

\begin{tabular}{|l|l|l|}
\hline Description & Value & Units \\
\hline Lime Solution Feed System & 559 & lb/d \\
\hline Quantities & 1120 & gpd(US) \\
\hline Lime feed rate of Ca(OH)2 & 1610 & pers-hrs/yr \\
\hline Liquid chemical solution fed & 673 & pers-hrs/yr \\
\hline O\&M labor required & 2280 & pers-hrs/yr \\
\hline Dry material handling and mixing labor required & \multicolumn{2}{|l|}{} \\
\hline Total operation labor required & 109000 & $\$$ \\
\hline Costs & \multicolumn{2}{|l|}{} \\
\hline Construction and equipment cost &
\end{tabular}

Table S31. Summary of Influent Pump Station for the retrofitting design with pyrolysis.

\begin{tabular}{|l|l|l|}
\hline Description & Value & Units \\
\hline Pump Station &
\end{tabular}




\begin{tabular}{|c|c|c|}
\hline \multicolumn{3}{|l|}{ Design Information } \\
\hline Volume of wet well & 19100 & cuft \\
\hline Width of wet well & 135 & $\mathrm{ft}$ \\
\hline Depth of the pumping station & 29.4 & $\mathrm{ft}$ \\
\hline Length of the pumping station & 32.2 & $\mathrm{ft}$ \\
\hline Width of the pumping station & 168 & $\mathrm{ft}$ \\
\hline Minimum depth of water in wet well & 8.43 & $\mathrm{ft}$ \\
\hline Area of pump building & 1100 & sqft \\
\hline Peak capacity of pumps & 39.9 & MGD(US) \\
\hline Firm pumping capacity & 39.9 & MGD(US) \\
\hline Total dynamic head - average conditions & 44 & $\mathrm{ft}$ \\
\hline \multicolumn{3}{|l|}{ Quantities } \\
\hline Operation labor required & 986 & pers-hrs/yr \\
\hline Maintenance labor required & 926 & pers-hrs/yr \\
\hline Electrical energy required & 807000 & $\mathrm{kWh} / \mathrm{yr}$ \\
\hline Volume of earthwork required & 612000 & cuft \\
\hline Volume of slab concrete required & 55800 & cuft \\
\hline Volume of wall concrete required & 16800 & cuft \\
\hline Capacity per pump & 13800 & gpm(US) \\
\hline Number of constant speed pumps & 3 & \\
\hline Number of variable speed pumps & 0 & \\
\hline Diameter of discharge header pipe & 37.6 & in \\
\hline Total dynamic head & 53.5 & $\mathrm{ft}$ \\
\hline Size of selected pump & 24 & in \\
\hline Specific speed of pump & 3570 & \\
\hline Pump rotating speed & 672 & rpm \\
\hline Motor size required & 194 & HP \\
\hline Size of selected motor & 200 & HP \\
\hline Width of pump system & 5.4 & $\mathrm{ft}$ \\
\hline
\end{tabular}




\begin{tabular}{|l|l|l|}
\hline Length of pump system & 23.5 & $\mathrm{ft}$ \\
\hline Length of the dry well & 32.2 & $\mathrm{ft}$ \\
\hline Width of the dry well & 32.5 & $\mathrm{ft}$ \\
\hline Costs & 2730000 & $\$$ \\
\hline Construction and equipment cost & 181000 & $\$$ \\
\hline Earthwork Cost & 405000 & $\$$ \\
\hline Wall Concrete Cost & 724000 & $\$$ \\
\hline Slab Concrete Cost & 121000 & $\$$ \\
\hline Building Cost & 880000 & $\$$ \\
\hline Installed Pump Equipment Cost & 416000 & $\$$ \\
\hline Misc Costs & & $\$$ \\
\hline
\end{tabular}

Table S32. Summary of Preliminary Treatment for the retrofitting design with pyrolysis.

\begin{tabular}{|l|l|l|}
\hline Description & Value & Units \\
\hline Preliminary Treatment & \multicolumn{2}{l|}{} \\
\hline Design Information & 0.25 & in \\
\hline Mechanically Cleaned Bar Screen & 1.5 & in \\
\hline Bar size & 30 & degrees \\
\hline Bar spacing & 0.0206 & $\mathrm{ft}$ \\
\hline Slope of bars from horizontal & 2.5 & $\mathrm{ft} / \mathrm{s}$ \\
\hline Head loss through screen & 2.5 & $\mathrm{ft} / \mathrm{s}$ \\
\hline Approach velocity & 3 & $\mathrm{ft} / \mathrm{s}$ \\
\hline Average flow through velocity (screen) & 8.07 & $\mathrm{ft}$ \\
\hline Maximum flow through velocity (screen) & 1 & $\mathrm{ft}$ \\
\hline Screen channel width & \multicolumn{2}{|l|}{} \\
\hline Average channel depth & 46.2 & $\mathrm{cuft} / \mathrm{s}$ \\
\hline Horizontal Flow Grit Chamber & 20.2 & $\mathrm{cuft} / \mathrm{s}$ \\
\hline Maximum flow & \multicolumn{2}{|l|}{} \\
\hline Average flow &
\end{tabular}




\begin{tabular}{|l|l|l|}
\hline Minimum flow & 15.4 & cuft/s \\
\hline Temperature & 10 & $\mathrm{deg}$ C \\
\hline Maximum flow through velocity (grit chamber) & 1.5 & $\mathrm{ft} / \mathrm{s}$ \\
\hline Average flow through velocity (grit chamber) & 1 & $\mathrm{ft} / \mathrm{s}$ \\
\hline Size of smallest particle 100\% removed & 0.2 & $\mathrm{~mm}$ \\
\hline Specific gravity of particle & 2.65 & \\
\hline Number of units & 2 & \\
\hline Maximum flow/unit & 23.1 & $\mathrm{cuft} / \mathrm{s}$ \\
\hline Width of channel & 3.85 & $\mathrm{ft}$ \\
\hline Depth of channel & 4 & $\mathrm{ft}$ \\
\hline Length of channel & 144 & $\mathrm{ft}$ \\
\hline Settling velocity of particle & 0.0707 & $\mathrm{ft} / \mathrm{s}$ \\
\hline Slope of channel bottom & 0.00048 & \\
\hline Allowance for currents & 1.7 & \\
\hline Manning coefficient & 0.035 & $\mathrm{~min}$ \\
\hline Hydraulic retention time & 1.6 & $\mathrm{cuft} / \mathrm{d}$ \\
\hline Volume of grit & 52.4 & \\
\hline Costs & 1390000 & $\mathbf{2}$ \\
\hline Construction and equipment cost & \multicolumn{2}{|l|}{} \\
\hline
\end{tabular}

Table S33. Summary of Upflow Anaerobic Sludge Blanket Reactor for the retrofitting design with pyrolysis.

\begin{tabular}{|l|l|l|}
\hline Description & Value & Units \\
\hline Upflow Anaerobic Sludge Blanket Reactor & \multicolumn{2}{l|}{} \\
\hline Design Information & 14.1 & $\mathrm{~d}$ \\
\hline Design SRT & 172000 & $\mathrm{~kg}$ \\
\hline Total reactor suspended solids & 0.822 & $\mathrm{mg}$ VSS/mg SS \\
\hline Calculated VSS:TSS ratio & 3.33 & $\mathrm{hr}$ \\
\hline Reactor hydraulic retention time &
\end{tabular}




\begin{tabular}{|c|c|c|}
\hline Total volume of reactors & 6890 & $\mathrm{~m} 3$ \\
\hline Total reactor surface area & 1380 & $\mathrm{~m} 2$ \\
\hline Reactor height & 5 & $\mathrm{~m}$ \\
\hline Upflow velocity & 1.5 & $\mathrm{~m} / \mathrm{hr}$ \\
\hline Sludge loading rate & 0.226 & $\mathrm{kgCOD} / \mathrm{kgVSS} / \mathrm{d}$ \\
\hline Calculated volumetric loading rate for this design & 5.64 & $\mathrm{~kg} / \mathrm{m} 3 / \mathrm{d}$ \\
\hline User-defined maximum loading rate & 18 & $\mathrm{~kg} / \mathrm{m} 3 / \mathrm{d}$ \\
\hline Calculated maximum loading rate & 23.4 & $\mathrm{~kg} / \mathrm{m} 3 / \mathrm{d}$ \\
\hline Methane production & 0.187 & m3 gas/m3 wastewater \\
\hline Potential energy gain from methane & 1160 & kW \\
\hline Effluent sulphide COD & 88.9 & $\mathrm{gCOD} / \mathrm{m} 3$ \\
\hline Amount of alkalinity required & 8.97 & meq \\
\hline Estimated alkalinity gained by acid reduction & 1.69 & meq \\
\hline Amount of sludge wasted & 12200 & $\mathrm{~kg} / \mathrm{d}$ \\
\hline Nitrogen requirement for biomass growth & 8.17 & $\mathrm{mg} / \mathrm{L}$ \\
\hline Phosphorus requirement for biomass growth & 2.04 & $\mathrm{mg} / \mathrm{L}$ \\
\hline \multicolumn{3}{|l|}{ Quantities } \\
\hline Operation labor required & 12500 & pers-hrs/yr \\
\hline Maintenance labor required & 1500 & pers-hrs/yr \\
\hline \multicolumn{3}{|l|}{ Costs } \\
\hline Construction and equipment cost & 5500000 & $\$$ \\
\hline \multicolumn{3}{|l|}{ Wastewater Pumping } \\
\hline \multicolumn{3}{|l|}{ Design Information } \\
\hline Average daily pumping rate & 13.1 & MGD(US) \\
\hline Total pumping capacity & 30 & MGD(US) \\
\hline Design capacity per pump & 10400 & gpm(US) \\
\hline Number of pumps & 3 & \\
\hline Number of batteries & 1 & \\
\hline Firm pumping capacity & 13.1 & MGD(US) \\
\hline
\end{tabular}




\begin{tabular}{|c|c|c|}
\hline \multicolumn{3}{|l|}{ Quantities } \\
\hline Operation labor required & 697 & pers-hrs/yr \\
\hline Maintenance labor required & 588 & pers-hrs/yr \\
\hline Electrical energy required & 436000 & $\mathrm{kWh} / \mathrm{yr}$ \\
\hline Volume of earthwork required & 6330 & cuft \\
\hline Area of pump building & 792 & sqft \\
\hline \multicolumn{3}{|l|}{ Costs } \\
\hline Construction and equipment cost & 572000 & $\$$ \\
\hline
\end{tabular}

Table S34. Summary of Counter Current Ammonia Stripping for the retrofitting design with pyrolysis.

\begin{tabular}{|l|l|l|}
\hline Description & Value & Units \\
\hline Counter Current Ammonia Stripping & \multicolumn{2}{l|}{} \\
\hline Design Information & \multicolumn{2}{l|}{} \\
\hline Liquid loading rate & 600 & $\mathrm{lb} /(\mathrm{sqft} \cdot \mathrm{hr})$ \\
\hline Gas loading rate & 1800 & $\mathrm{lb} /(\mathrm{sqft} \cdot \mathrm{hr})$ \\
\hline Tower surface area & 194 & $\mathrm{sqft}$ \\
\hline Tower height & 32.2 & $\mathrm{ft}$ \\
\hline Initial ammonia concentration & 78.6 & $\mathrm{mg} / \mathrm{L}$ \\
\hline Effluent ammonia concentration & 2 & $\mathrm{mg} / \mathrm{L}$ \\
\hline Waste water flow & 0.335 & $\mathrm{MGD}(\mathrm{US})$ \\
\hline Lime required & 559 & $\mathrm{lb} / \mathrm{d}$ \\
\hline Costs & \multicolumn{2}{|l}{} \\
\hline Construction and equipment cost & 252000 & $\$$ \\
\hline
\end{tabular}

Table S35. Summary of Anaerobic Digestion for the retrofitting design with pyrolysis.

\begin{tabular}{|l|l|l|}
\hline Description & Value & Units \\
\hline Anaerobic Digestion & $\%$ \\
\hline Design Information & 50 & $\%$ \\
\hline Percent VSS destroyed &
\end{tabular}




\begin{tabular}{|c|c|c|}
\hline Solids concentration in digester & 5 & $\%$ \\
\hline Detention time & 28 & $\mathrm{~d}$ \\
\hline Digester depth & 28.8 & $\mathrm{ft}$ \\
\hline Digester diameter & 75 & $\mathrm{ft}$ \\
\hline Effective digester volume & 282000 & cuft \\
\hline Number of digesters per battery & 2 & \\
\hline Number of primary digesters per battery & 1 & \\
\hline Number of secondary digesters per battery & 1 & \\
\hline Number of batteries & 1 & \\
\hline Gas produced & 38.1 & cuft/min \\
\hline Heat required & 1290000 & BTU/hr \\
\hline Digester gas required & 49.9 & cuft/min \\
\hline Total natural gas required & 1230000 & $\mathrm{cuft} / \mathrm{yr}$ \\
\hline \multicolumn{3}{|l|}{ Quantities } \\
\hline Operation labor required & 1760 & pers-hrs/yr \\
\hline Maintenance labor required & 1160 & pers-hrs/yr \\
\hline Electrical energy required & 156000 & $\mathrm{kWh} / \mathrm{yr}$ \\
\hline Volume of earthwork required & 281000 & cuft \\
\hline Slab thickness & 11.2 & in \\
\hline Volume of slab concrete required & 8980 & cuft \\
\hline Wall thickness & 21.9 & in \\
\hline Volume of wall concrete required & 30100 & cuft \\
\hline Sidewater depth & 28.8 & $\mathrm{ft}$ \\
\hline Surface area/floor of 2-story control bldg & 1650 & sqft \\
\hline Piping size & 10 & in \\
\hline Length of total piping system & 694 & $\mathrm{ft}$ \\
\hline Number of 90 degree elbows & 26 & \\
\hline Number of tees & 51 & \\
\hline Number of plug valves & 37 & \\
\hline
\end{tabular}




\begin{tabular}{|l|l|l|}
\hline Total dry solids treated & 7.53 & ton(short)/d \\
\hline Costs & 4780000 & $\$$ \\
\hline Construction and equipment cost & \multicolumn{2}{|l|}{} \\
\hline
\end{tabular}

Table S36. Summary of Plug Flow Activated Sludge for the retrofitting design with pyrolysis.

\begin{tabular}{|c|c|c|}
\hline Description & Value & Units \\
\hline \multicolumn{3}{|l|}{ Plug-Flow Aerated Bioreactor } \\
\hline \multicolumn{3}{|l|}{ Design Information } \\
\hline \multicolumn{3}{|l|}{ Carbon Only Design } \\
\hline Design SRT for design at winter temperature & 15 & $\mathrm{~d}$ \\
\hline Design SS & 2500 & $\mathrm{mg} / \mathrm{L}$ \\
\hline Calculated VSS & 1700 & $\mathrm{mg} / \mathrm{L}$ \\
\hline Calculated VSS:TSS ratio & 0.678 & mg VSS/mg SS \\
\hline Total volume of reactors & 30600 & $\mathrm{~m} 3$ \\
\hline Length of parallel train & 102 & $\mathrm{~m}$ \\
\hline Width of parallel train & 10 & $\mathrm{~m}$ \\
\hline Sidewater depth & 5 & $\mathrm{~m}$ \\
\hline Number of batteries & 1 & \\
\hline Number of parallel trains per battery & 6 & \\
\hline Number of cells within one train & 3 & \\
\hline Total number of dividing walls between zones & 12 & \\
\hline Hydraulic retention time & 14.7 & $\mathrm{hr}$ \\
\hline F/M ratio & 0.0646 & $\mathrm{~kg} \mathrm{BOD} / \mathrm{kg}$ MLSS/d \\
\hline Volumetric BOD loading & 0.109 & $\mathrm{~kg} \mathrm{BOD} / \mathrm{m} 3 / \mathrm{d}$ \\
\hline Observed yield (VSS basis) & 0.441 & g VSS/g BOD \\
\hline Observed yield (TSS basis) & 0.434 & g TSS/g BOD \\
\hline Amount of alkalinity required & 0 & gCaCO3/m3 \\
\hline Amount of sludge generated & 5100 & $\mathrm{~kg} / \mathrm{d}$ \\
\hline Sludge recycle rate & 16700 & $\mathrm{~m} 3 / \mathrm{d}$ \\
\hline
\end{tabular}




\begin{tabular}{|c|c|c|}
\hline Nitrogen requirement for biomass growth & 4.64 & $\mathrm{mg} / \mathrm{L}$ \\
\hline Phosphorus requirement for biomass growth & 0.928 & $\mathrm{mg} / \mathrm{L}$ \\
\hline Oxygen requirement to meet average demand & 14300 & $\mathrm{~kg} / \mathrm{d}$ \\
\hline Air flow required to meet average demand & 34500 & $\mathrm{~N} \mathrm{~m} 3 / \mathrm{hr}$ \\
\hline Design air flow & 18.8 & $\mathrm{~N} \mathrm{~m} 3 / \mathrm{min} / 1000 \mathrm{~m} 3$ \\
\hline \multicolumn{3}{|l|}{ Quantities } \\
\hline Operation labor required & 3930 & pers-hrs/yr \\
\hline Maintenance labor required & 2410 & pers-hrs/yr \\
\hline Electrical energy required & 5240000 & $\mathrm{kWh} / \mathrm{yr}$ \\
\hline Volume of earthwork required & 504000 & cuft \\
\hline Volume of slab concrete required & 159000 & cuft \\
\hline Volume of wall concrete required & 97800 & cuft \\
\hline Handrail length & 3620 & $\mathrm{ft}$ \\
\hline Number of diffusers per train & 1700 & \\
\hline Fine bubble diffuser floor coverage & 6.82 & $\%$ \\
\hline Number of swing arm headers per train & 14 & \\
\hline \multicolumn{3}{|l|}{ Costs } \\
\hline Construction and equipment cost & 9320000 & $\$$ \\
\hline Earthwork Cost & 149000 & $\$$ \\
\hline Wall Concrete Cost & 2360000 & $\$$ \\
\hline Slab Concrete Cost & 2060000 & $\$$ \\
\hline Handrail Cost & 272000 & $\$$ \\
\hline Installed Aerator Equipment Cost & 2460000 & $\$$ \\
\hline Air Piping Cost & 1090000 & $\$$ \\
\hline Misc Costs & 923000 & $\$$ \\
\hline \multicolumn{3}{|l|}{ Sludge Recycle Pumping } \\
\hline \multicolumn{3}{|l|}{ Design Information } \\
\hline Average daily pumping rate & 4.41 & MGD(US) \\
\hline Total pumping capacity & 8.82 & MGD(US) \\
\hline
\end{tabular}




\begin{tabular}{|l|l|l|}
\hline Design capacity per pump & 3060 & gpm(US) \\
\hline Number of pumps & 3 & \\
\hline Number of batteries & 1 & \\
\hline Firm pumping capacity & 4.41 & MGD(US) \\
\hline Quantities & \multicolumn{2}{|l|}{} \\
\hline Operation labor required & 532 & pers-hrs/yr \\
\hline Maintenance labor required & 448 & pers-hrs/yr \\
\hline Electrical energy required & 147000 & kWh/yr \\
\hline Volume of earthwork required & 2990 & cuft \\
\hline Area of pump building & 374 & sqft \\
\hline Costs & & \\
\hline Construction and equipment cost & 210000 & $\$$ \\
\hline Earthwork Cost & 886 & $\$$ \\
\hline Pump Building Cost & 41100 & $\$$ \\
\hline Installed Pump Cost & 136000 & $\$$ \\
\hline Misc Costs & 32000 & $\$$ \\
\hline
\end{tabular}

Table S37. Summary of Gravity Thickening for the retrofitting design with pyrolysis.

\begin{tabular}{|l|l|l|}
\hline Description & Value & Units \\
\hline Gravity Thickening & \multicolumn{2}{l|}{} \\
\hline Design Information & 1.35 & $\%$ \\
\hline Initial concentration & 5 & $\%$ \\
\hline Thickened concentration & 10 & $\mathrm{lb} /(\mathrm{sqft} \cdot \mathrm{d})$ \\
\hline Mass loading & 88.6 & $\mathrm{gal}(\mathrm{US}) /(\mathrm{sqft} \cdot \mathrm{d})$ \\
\hline Hydraulic loading & 18.2 & $\mathrm{hr}$ \\
\hline Hydraulic retention time & 2 & \\
\hline Number of tanks & 15100 & $\mathrm{cuft}$ \\
\hline Tank volume & 9 & $\mathrm{ft}$ \\
\hline Depth & &
\end{tabular}




\begin{tabular}{|c|c|c|}
\hline Surface area per tank & 836 & sqft \\
\hline Tank diameter & 33 & $\mathrm{ft}$ \\
\hline \multicolumn{3}{|l|}{ Quantities } \\
\hline Amount of sludge generated & 8.36 & ton(short)/d \\
\hline Volume of thickened sludge & 34400 & gpd(US) \\
\hline Operation labor required & 538 & pers-hrs/yr \\
\hline Maintenance labor required & 393 & pers-hrs/yr \\
\hline Electrical energy required & 8530 & $\mathrm{kWh} / \mathrm{yr}$ \\
\hline Volume of earthwork required & 21800 & cuft \\
\hline Slab thickness & 10.1 & in \\
\hline Volume of slab concrete required & 1910 & cuft \\
\hline Wall thickness & 11.5 & in \\
\hline Volume of wall concrete required & 2300 & cuft \\
\hline \multicolumn{3}{|l|}{ Costs } \\
\hline Construction and equipment cost & 307000 & $\$$ \\
\hline Earthwork Cost & 6450 & $\$$ \\
\hline Wall Concrete Cost & 55500 & $\$$ \\
\hline Slab Concrete Cost & 24800 & $\$$ \\
\hline Installed Equipment Cost & 174000 & $\$$ \\
\hline Misc Costs & 46900 & $\$$ \\
\hline
\end{tabular}

Table S38. Summary of Belt-Filter Press for the retrofitting design with pyrolysis.

\begin{tabular}{|l|l|l|}
\hline Description & Value & Units \\
\hline Belt-Filter Press & 2 & \\
\hline Design Information & \multicolumn{2}{l|}{} \\
\hline Belt filter width & 8 & m \\
\hline Number of units & 70 & \\
\hline Hydraulic loading per unit per meter of belt width & 70 & gpm(US) \\
\hline Hydraulic loading required per meter of belt width & 1020 & gpm(US) \\
\hline
\end{tabular}




\begin{tabular}{|l|l|l|}
\hline Final solids content & 20 & $\%$ \\
\hline Solids capture fraction & 0.992 & \multicolumn{2}{l|}{} \\
\hline Quantities & 1260 & pers-hrs/yr \\
\hline Operation labor required & 315 & pers-hrs/yr \\
\hline Maintenance labor required & 333000 & $\mathrm{kWh} / \mathrm{yr}$ \\
\hline Power & 165000 & $\mathrm{lb} / \mathrm{yr}$ \\
\hline Polymer required & 45100 & $\mathrm{lb} / \mathrm{d}$ \\
\hline Dry solids produced & 2540000 & $\$$ \\
\hline Belt filter(s) & 572000 & $\$$ \\
\hline Building & 636000 & $\$$ \\
\hline Installation & 941000 & $\$$ \\
\hline Polymer system & 280000 & $\$$ \\
\hline Feed pumps & 636000 & $\$$ \\
\hline Conveyor system & & \\
\hline Costs & 5610000 & $\$$ \\
\hline Construction and equipment cost & 572000 & $\$$ \\
\hline Building Cost & 941000 & $\$$ \\
\hline Polymer System Cost & 280000 & $\$$ \\
\hline Feed Pumps Cost & 636000 & $\$$ \\
\hline Conveyor System Cost & 3180000 & $\$$ \\
\hline Installed Belt Filter & & \\
\hline
\end{tabular}

Table S39. Summary of Secondary Clarifier for the retrofitting design with pyrolysis.

\begin{tabular}{|l|l|l|}
\hline Description & Value & Units \\
\hline Secondary Clarification & 60300 & sqft \\
\hline Design Information & 15100 & sqft \\
\hline Surface area & 139 & $\mathrm{ft}$ \\
\hline Surface area per circular clarifier & & \multicolumn{2}{|l|}{} \\
\hline Diameter of each circular clarifier &
\end{tabular}




\begin{tabular}{|c|c|c|}
\hline Number of clarifiers per battery & 4 & \\
\hline Number of batteries & 1 & \\
\hline Solids loading rate & 6.1 & $\mathrm{lb} /(\mathrm{sqft} \cdot \mathrm{d})$ \\
\hline Hydraulic retention time & 7.36 & $\mathrm{hr}$ \\
\hline Designed surface overflow rate & 500 & gal(US)/(sqft·d) \\
\hline Weir length & 2010 & $\mathrm{ft}$ \\
\hline Volume of wasted sludge & 131000 & gpd(US) \\
\hline \multicolumn{3}{|l|}{ Quantities } \\
\hline Operation labor required & 3020 & pers-hrs/yr \\
\hline Maintenance labor required & 1680 & pers-hrs/yr \\
\hline Electrical energy required & 23200 & $\mathrm{kWh} / \mathrm{yr}$ \\
\hline Volume of earthwork required & 917000 & cuft \\
\hline Slab thickness & 10.1 & in \\
\hline Volume of slab concrete required & 57100 & cuft \\
\hline Wall thickness & 11.5 & in \\
\hline Volume of wall concrete required & 18000 & cuft \\
\hline \multicolumn{3}{|l|}{ Costs } \\
\hline Construction and equipment cost & 2690000 & $\$$ \\
\hline Earthwork Cost & 272000 & $\$$ \\
\hline Wall Concrete Cost & 434000 & $\$$ \\
\hline Slab Concrete Cost & 740000 & $\$$ \\
\hline Installed Equipment Cost & 837000 & $\$$ \\
\hline Misc Costs & 411000 & $\$$ \\
\hline \multicolumn{3}{|l|}{ Waste Sludge Pumping } \\
\hline \multicolumn{3}{|l|}{ Design Information } \\
\hline Average daily pumping rate & 0.131 & MGD(US) \\
\hline Total pumping capacity & 0.131 & MGD(US) \\
\hline Design capacity per pump & 45.4 & gpm(US) \\
\hline Number of pumps & 3 & \\
\hline
\end{tabular}




\begin{tabular}{|l|l|l|}
\hline Number of batteries & 1 & \\
\hline Firm pumping capacity & 0.131 & MGD(US) \\
\hline Quantities & \multicolumn{2}{|l|}{} \\
\hline Operation labor required & 339 & pers-hrs/yr \\
\hline Maintenance labor required & 267 & pers-hrs/yr \\
\hline Electrical energy required & 4400 & $\mathrm{kWh} / \mathrm{yr}$ \\
\hline Volume of earthwork required & 1620 & cuft \\
\hline Area of pump building & 203 & sqft \\
\hline Costs & \multicolumn{2}{|l|}{} \\
\hline Construction and equipment cost & 51900 & $\$$ \\
\hline Earthwork Cost & 480 & $\$$ \\
\hline Pump Building Cost & 22300 & $\$$ \\
\hline Installed Pump Cost & 21200 & $\$$ \\
\hline Misc Costs & 7920 & $\$$ \\
\hline
\end{tabular}

Table S40. Summary of Chlorination for the retrofitting design with pyrolysis.

\begin{tabular}{|l|l|l|}
\hline Description & Value & Units \\
\hline Chlorination & \multicolumn{2}{l|}{} \\
\hline Design Information & 625000 & gal(US) \\
\hline Volume of tank & 1090 & $\mathrm{lb} / \mathrm{d}$ \\
\hline Average chlorine required & 2500 & $\mathrm{lb} / \mathrm{d}$ \\
\hline Peak chlorine required & 10000000 & $/ 100 \mathrm{ml}$ \\
\hline Influent coliform count & 29.2 & $/ 100 \mathrm{ml}$ \\
\hline Effluent coliform count & \multicolumn{2}{|l|}{} \\
\hline Quantities & 1670 & pers-hrs/yr \\
\hline Operational labor required & 471 & pers-hrs/yr \\
\hline Maintenance labor required & 138000 & $\mathrm{kWh} / \mathrm{yr}$ \\
\hline Electrical energy required & 35500 & cuft \\
\hline Volume of earthwork required & &
\end{tabular}




\begin{tabular}{|l|l|l|}
\hline Volume of slab concrete required & 8370 & cuft \\
\hline Volume of wall concrete required & 11300 & cuft \\
\hline Number of chlorinators and evaporators & 1 & \\
\hline Chlorination building area & 220 & sqft \\
\hline Number of chlorine cylinders & 17 & \\
\hline Area of chlorine storage building & 2380 & sqft \\
\hline Costs & \multicolumn{2}{|l|}{} \\
\hline Construction and equipment cost & 1040000 & $\$$ \\
\hline Earthwork Cost & 10500 & $\$$ \\
\hline Wall Concrete Cost & 272000 & $\$$ \\
\hline Slab Concrete Cost & 108000 & $\$$ \\
\hline Installed Equipment Cost & 422000 & $\$$ \\
\hline Building Cost & 24200 & $\$$ \\
\hline Storage Building Cost & 131000 & $\$$ \\
\hline Misc Costs & 70400 & $\$$ \\
\hline
\end{tabular}

\section{S5. NPV calculations}

Table S41. NPV calculation for the reference design.

\begin{tabular}{|c|c|c|c|}
\hline & $\begin{array}{l}\text { Direct manufacturing costs } \\
\text { (feed, utilities, labor, maintenance) }\end{array}$ & $\$$ & $9,212,362.95$ \\
\hline \multirow{4}{*}{$\mathrm{C}$} & Operating overhead & $\$$ & $976,187.96$ \\
\hline & $\begin{array}{l}\text { Fixed costs (property } \\
\text { insurance, depreciation) }\end{array}$ & $\$$ & 2,318,613.64 \\
\hline & General expense & $\$$ & $37,953.23$ \\
\hline & Subtotal & $\$$ & $12,545,117.78$ \\
\hline $\mathrm{S}$ & Annual sales & $\$$ & $33,846,971.26$ \\
\hline \multirow[t]{2}{*}{$\mathrm{t}$} & Tax & & 0.4 \\
\hline & Plant construction year & $\$$ & $(16,024,196.49)$ \\
\hline \multirow[t]{2}{*}{ CF } & Plant operation startup year & $\$$ & 8,916,756.02 \\
\hline & Plant operation years & $\$$ & $14,069,230.78$ \\
\hline \multicolumn{4}{|c|}{ year } \\
\hline 0 & & $\$$ & $(16,024,196.49)$ \\
\hline 1 & & $\$$ & 8,916,756.02 \\
\hline
\end{tabular}




\begin{tabular}{lll}
2 & $\$$ & $14,069,230.78$ \\
3 & $\$$ & $14,069,230.78$ \\
4 & $\$$ & $14,069,230.78$ \\
5 & $\$$ & $14,069,230.78$ \\
6 & $\$$ & $14,069,230.78$ \\
7 & $\$$ & $14,069,230.78$ \\
8 & $\$$ & $14,069,230.78$ \\
9 & $\$$ & $14,069,230.78$ \\
10 & $\$$ & $14,069,230.78$ \\
11 & $\$$ & $14,069,230.78$ \\
12 & $\$$ & $14,069,230.78$ \\
13 & $\$$ & $14,069,230.78$ \\
14 & $\$$ & $14,069,230.78$ \\
15 & $\$$ & $14,069,230.78$ \\
16 & $\$$ & $14,069,230.78$ \\
17 & $\$$ & $14,069,230.78$ \\
18 & $\$$ & $14,069,230.78$ \\
19 & $\$$ & $14,069,230.78$ \\
20 & $\$$ & $14,069,230.78$ \\
\hline NPV & $\$$ & $154,402,397.99$ \\
\hline
\end{tabular}

Table S42. NPV calculation for the retrofitting design with HTL.

\begin{tabular}{|c|c|c|c|}
\hline \multirow{5}{*}{$\mathrm{C}$} & $\begin{array}{l}\text { Direct manufacturing costs } \\
\text { (feed, utilities, labor, maintenance) }\end{array}$ & $\$$ & $10,234,426.47$ \\
\hline & Operating overhead & $\$$ & $1,134,549.44$ \\
\hline & $\begin{array}{l}\text { Fixed costs (property } \\
\text { insurance, depreciation) }\end{array}$ & $\$$ & 2,961,494.53 \\
\hline & General expense & $\$$ & $100,484.43$ \\
\hline & Subtotal & $\$$ & $14,430,954.88$ \\
\hline S & Annual sales & $\$$ & $38,983,384.99$ \\
\hline $\mathrm{t}$ & Tax & & 0.4 \\
\hline \multirow{3}{*}{$\mathrm{CF}$} & Plant construction year & $\$$ & $(20,467,217.77)$ \\
\hline & Plant operation startup year & $\$$ & 9,795,633.84 \\
\hline & Plant operation years & $\$$ & $16,376,732.80$ \\
\hline \multicolumn{4}{|c|}{ year } \\
\hline 0 & & $\$$ & $(20,467,217.77)$ \\
\hline 1 & & $\$$ & $9,795,633.84$ \\
\hline 2 & & $\$$ & $16,376,732.80$ \\
\hline 3 & & $\$$ & $16,376,732.80$ \\
\hline 4 & & $\$$ & $16,376,732.80$ \\
\hline 5 & & $\$$ & $16,376,732.80$ \\
\hline
\end{tabular}




\begin{tabular}{lll}
6 & $\$$ & $16,376,732.80$ \\
7 & $\$$ & $16,376,732.80$ \\
8 & $\$$ & $16,376,732.80$ \\
9 & $\$$ & $16,376,732.80$ \\
10 & $\$$ & $16,376,732.80$ \\
11 & $\$$ & $16,376,732.80$ \\
12 & $\$$ & $16,376,732.80$ \\
13 & $\$$ & $16,376,732.80$ \\
14 & $\$$ & $16,376,732.80$ \\
15 & $\$$ & $16,376,732.80$ \\
16 & $\$$ & $16,376,732.80$ \\
17 & $\$$ & $16,376,732.80$ \\
18 & $\$$ & $16,376,732.80$ \\
19 & $\$$ & $16,376,732.80$ \\
20 & $\$$ & $16,376,732.80$ \\
\hline NPV & $\$$ & $177,355,357.87$ \\
\hline
\end{tabular}

Table S43. NPV calculation for the retrofitting design with slow pyrolysis.

\begin{tabular}{|c|c|c|c|}
\hline \multirow{5}{*}{$\mathrm{C}$} & $\begin{array}{l}\text { Direct manufacturing costs } \\
\text { (feed, utilities, labor, maintenance) }\end{array}$ & $\$$ & $16,097,655.70$ \\
\hline & Operating overhead & $\$$ & $1,280,293.80$ \\
\hline & $\begin{array}{l}\text { Fixed costs (property } \\
\text { insurance, depreciation) }\end{array}$ & $\$$ & 3,122,029.33 \\
\hline & General expense & $\$$ & $63,833.85$ \\
\hline & Subtotal & $\$$ & $20,563,812.68$ \\
\hline$S$ & Annual sales & $\$$ & $34,970,124.21$ \\
\hline $\mathrm{t}$ & Tax & & 0.4 \\
\hline \multirow{3}{*}{$\mathrm{CF}$} & Plant construction year & $\$$ & $(21,576,691.60)$ \\
\hline & Plant operation startup year & $\$$ & $3,440,404.70$ \\
\hline & Plant operation years & $\$$ & $10,378,247.66$ \\
\hline \multicolumn{4}{|c|}{ year } \\
\hline 0 & & $\$$ & $(21,576,691.60)$ \\
\hline 1 & & $\$$ & $3,440,404.70$ \\
\hline 2 & & $\$$ & $10,378,247.66$ \\
\hline 3 & & $\$$ & $10,378,247.66$ \\
\hline 4 & & $\$$ & $10,378,247.66$ \\
\hline 5 & & $\$$ & $10,378,247.66$ \\
\hline 6 & & $\$$ & $10,378,247.66$ \\
\hline 7 & & $\$$ & $10,378,247.66$ \\
\hline 8 & & $\$$ & $10,378,247.66$ \\
\hline 9 & & $\$$ & $10,378,247.66$ \\
\hline
\end{tabular}




\begin{tabular}{lrr}
10 & $\$$ & $10,378,247.66$ \\
11 & $\$$ & $10,378,247.66$ \\
12 & $\$$ & $10,378,247.66$ \\
13 & $\$$ & $10,378,247.66$ \\
14 & $\$$ & $10,378,247.66$ \\
15 & $\$$ & $10,378,247.66$ \\
16 & $\$$ & $10,378,247.66$ \\
17 & $\$$ & $10,378,247.66$ \\
18 & $\$$ & $10,378,247.66$ \\
19 & $\$$ & $10,378,247.66$ \\
20 & $\$$ & $10,378,247.66$ \\
\hline NPV & $\$$ & $101,151,744.24$ \\
\hline
\end{tabular}

Table S44. NPV calculation for the retrofitting design with fast pyrolysis.

\begin{tabular}{|c|c|c|c|}
\hline \multirow{5}{*}{$\mathrm{C}$} & $\begin{array}{l}\text { Direct manufacturing costs } \\
\text { (feed, utilities, labor, maintenance) }\end{array}$ & $\$$ & $16,081,363.44$ \\
\hline & Operating overhead & $\$$ & $1,290,092.89$ \\
\hline & $\begin{array}{l}\text { Fixed costs (property } \\
\text { insurance, depreciation) }\end{array}$ & $\$$ & $3,049,949.26$ \\
\hline & General expense & $\$$ & $145,947.39$ \\
\hline & Subtotal & $\$$ & $20,567,352.98$ \\
\hline S & Annual sales & $\$$ & $35,247,525.17$ \\
\hline $\mathrm{t}$ & Tax & & 0.4 \\
\hline \multirow{3}{*}{$\mathrm{CF}$} & Plant construction year & $\$$ & $(21,078,538.23)$ \\
\hline & Plant operation startup year & $\$$ & $3,724,854.54$ \\
\hline & Plant operation years & $\$$ & $10,502,519.57$ \\
\hline \multicolumn{4}{|c|}{ year } \\
\hline 0 & & $\$$ & $(21,078,538.23)$ \\
\hline 1 & & $\$$ & 3,724,854.54 \\
\hline 2 & & $\$$ & $10,502,519.57$ \\
\hline 3 & & $\$$ & $10,502,519.57$ \\
\hline 4 & & $\$$ & $10,502,519.57$ \\
\hline 5 & & $\$$ & $10,502,519.57$ \\
\hline 6 & & $\$$ & $10,502,519.57$ \\
\hline 7 & & $\$$ & $10,502,519.57$ \\
\hline 8 & & $\$$ & $10,502,519.57$ \\
\hline 9 & & $\$$ & $10,502,519.57$ \\
\hline 10 & & $\$$ & $10,502,519.57$ \\
\hline 11 & & $\$$ & $10,502,519.57$ \\
\hline 12 & & $\$$ & $10,502,519.57$ \\
\hline 13 & & $\$$ & $10,502,519.57$ \\
\hline
\end{tabular}




\begin{tabular}{lll}
14 & $\$$ & $10,502,519.57$ \\
15 & $\$$ & $10,502,519.57$ \\
16 & $\$$ & $10,502,519.57$ \\
17 & $\$$ & $10,502,519.57$ \\
18 & $\$$ & $10,502,519.57$ \\
19 & $\$$ & $10,502,519.57$ \\
20 & $\$$ & $10,502,519.57$ \\
\hline NPV & $\$$ & $103,351,150.72$ \\
\hline
\end{tabular}

